\title{
Modeling of the acoustic radiation force in elastography
}

Fabrice Prieur, and Oleg A. Sapozhnikov

Citation: The Journal of the Acoustical Society of America 142, 947 (2017); doi: 10.1121/1.4998585

View online: https://doi.org/10.1121/1.4998585

View Table of Contents: http://asa.scitation.org/toc/jas/142/2

Published by the Acoustical Society of America

\section{Articles you may be interested in}

Space-time domain solutions of the wave equation by a non-singular boundary integral method and Fourier transform

The Journal of the Acoustical Society of America 142, 697 (2017); 10.1121/1.4996860

Calibration of focused ultrasonic transducers and absolute measurements of fluid nonlinearity with diffraction and attenuation corrections

The Journal of the Acoustical Society of America 142, 984 (2017); 10.1121/1.4999328

Propagation of coherent transverse waves: Influence of the translational and rotational subwavelength resonances

The Journal of the Acoustical Society of America 142, 512 (2017); 10.1121/1.4996129

Instantaneous Bayesian regularization applied to real-time near-field acoustic holography

The Journal of the Acoustical Society of America 142, 924 (2017); 10.1121/1.4998571

Uniform theory of diffraction (UTD)-based solution for sound diffraction caused by an array of obstacles

The Journal of the Acoustical Society of America 142, 902 (2017); 10.1121/1.4997942

Ultrasonic harmonic generation from materials with up to cubic nonlinearity

The Journal of the Acoustical Society of America 142, EL224 (2017); 10.1121/1.4998139 


\title{
Modeling of the acoustic radiation force in elastography
}

\author{
Fabrice Prieur ${ }^{\mathrm{a})}$ \\ Department of Informatics, University of Oslo, Postboks 1080 Blindern, 0316 Oslo, Norway \\ Oleg A. Sapozhnikov ${ }^{\text {b) }}$ \\ Physics Faculty, M. V. Lomonosov Moscow State University, 1-2 Leninskiye Gory, Moscow GSP-1, Russia
}

(Received 2 May 2017; revised 14 July 2017; accepted 24 July 2017; published online 16 August 2017)

Elastography is a non-invasive imaging technique that can assess in vivo tissue stiffness. In shear wave elastography imaging, the acoustic radiation force (ARF) produced by focused ultrasound generates a local force that produces shear waves. The authors compare three existing formulations for the ARF: its full expression in the second-order approximation and two simplified formulations using a quasi-plane wave and an attenuated plane wave approximation. Analytical expressions for the ARF are derived for the special cases of a concave spherical source and a quasi-Gaussian beam. They provide expressions for the resulting ARF and show discrepancies between the different formulations. For strongly divergent or highly focused beams the ARF expressed by the second-order approximation significantly differs from both simplified formulations. However, despite those differences the second-order and quasi-plane wave approximations create identical shear displacements in tissue. To compute the ARF and the displacements produced by a conventional ultrasound probe, the three formulations were incorporated into the k-Wave simulation package. The secondorder and quasi-plane wave approximations give different forces but nearly identical displacements while the plane wave approximation significantly differs. It is concluded that to properly take into account the ultrasound field structure, the second-order or quasi-plane wave approximations should be preferably used. @ 2017 Acoustical Society of America. [http://dx.doi.org/10.1121/1.4998585]

[PLM]

Pages: 947-961

\section{INTRODUCTION}

Elastography is a technique now widely used to image the elastic properties of tissue. ${ }^{1,2}$ These properties are valuable for the diagnostics of pathologies such as cancer. Indeed the mechanical properties of such pathologic tissue vary from those of healthy tissue. ${ }^{3,4}$ Mapping of the local tissue elasticity inside the body can help reveal the presence of a tumor when other diagnostic modalities cannot.

Various techniques exist to obtain a local elasticity map. ${ }^{1}$ In this article we look at the shear wave elasticity imaging (SWEI) technique which uses the radiation force created by ultrasound to trigger a remote displacement in the body. ${ }^{5}$ This displacement generates the propagation of shear waves whose speed is directly linked to the elastic properties of the medium.

In this context, numerical simulation is a valuable tool to predict the amplitude and shape of the displacements that will propagate due to the acoustic radiation force (ARF) ${ }^{6-9}$ This involves modeling of the ARF generated by ultrasound and of the reaction of the medium to this force with the propagation of shear waves.

Our study looks at the existing formulations for the ARF generated by an ultrasound field, the spatial distribution of the force, and the displacement they create in the medium.

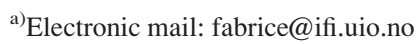

${ }^{b)}$ Also at Center for Industrial and Medical Ultrasound, Applied Physics Laboratory, University of Washington, 1013 NE 40th Street, Seattle, WA 98105, USA.
}

The goal is to compare the results and try to establish a practical domain of validity for these formulations.

We first present three distinct formulations for the ARF and the assumptions behind them. An analytical expression for the ARF is then derived in the case of a spherical concave source and a "quasi-Gaussian beam." Numerical simulations then show the spatial distribution of the force when using each formulation. The obtained distributions of ARF are subsequently used to simulate the ensuing displacements in the medium and their propagation.

\section{FORMULATIONS OF THE ARF}

\section{A. Generic expression}

We first recall the derivation for a general formulation for the ARF. The conservation of momentum for a particle in an inviscid fluid is written using Euler's equation ${ }^{10}$

$$
\rho \frac{d \mathbf{v}}{d t}=\rho\left[\frac{\partial \mathbf{v}}{\partial t}+(\mathbf{v} \cdot \nabla) \mathbf{v}\right]=-\nabla p
$$

where $\rho, \mathbf{v}$, and $p$ are the medium density, the particle velocity, and the pressure, respectively.

Combining it with the equation of continuity

$$
\frac{\partial \rho}{\partial t}+\nabla \cdot(\rho \mathbf{v})=0
$$

the rate of change of momentum in a fixed volume element can be written ${ }^{10}$ 


$$
\frac{\partial\left(\rho v_{i}\right)}{\partial t}=-\frac{\partial \Pi_{i k}}{\partial x_{k}}
$$

where the tensor notation was used and $k$ is the summation index. $\Pi_{i j}$ is the momentum flux density tensor defined as ${ }^{10}$

$$
\Pi_{i j}=p \delta_{i j}+\rho v_{i} v_{j},
$$

where $\delta_{i j}$ is the Kronecker delta.

As the motion of a wave is oscillatory a time average over a wave cycle for Eq. (3) gives the expression for the $\mathrm{ARF}^{11,12}$

$$
F_{i}=\left\langle\frac{\partial\left(\rho v_{i}\right)}{\partial t}\right\rangle=-\frac{\partial\left\langle\Pi_{i k}\right\rangle}{\partial x_{k}},
$$

where $F_{i}$ designates the components of the ARF.

Using Eq. (4) and introducing the equilibrium pressure $p_{0}$ which is constant we can write

$$
F_{i}=-\frac{\partial}{\partial x_{k}}\left(\left\langle p_{E}\right\rangle \delta_{i k}+\left\langle\rho v_{i} v_{k}\right\rangle\right),
$$

where $\left\langle p_{E}\right\rangle=\left\langle p-p_{0}\right\rangle$ is called the mean Eulerian excess pressure $^{11}$ and $\left\langle\rho v_{i} v_{j}\right\rangle$ is called the Reynolds stress sensor. This expression for the force applied to a fixed unit volume expressed in $\mathrm{N} / \mathrm{m}^{3}$ shows two distinct contributions. The first contribution comes from the pressure applied on the volume walls $\left(\left\langle p_{E}\right\rangle \delta_{i k}\right)$. The second comes from the net influx of momentum that penetrates into the volume through the volume walls due to the movement of the fluid particles $\left(\left\langle\rho v_{i} v_{k}\right\rangle\right)$.

\section{B. Second-order approximation}

Using a perturbation analysis, one can define

$$
\begin{aligned}
& \rho=\rho_{0}+\rho_{1}+\rho_{2}+\cdots, \\
& \mathbf{v}=\mathbf{v}_{1}+\mathbf{v}_{2}+\cdots, \\
& p=p_{0}+p_{1}+p_{2}+\cdots,
\end{aligned}
$$

where the subscripts 0,1 , and 2 refer to undisturbed values, the first-order, and the second-order small quantities, respectively. ${ }^{13,14}$

To the second order, one can show that the mean Eulerian excess pressure can be written using first-order quantities only $^{11,15}$

$$
\left\langle p_{E}\right\rangle=\frac{1}{2 \rho_{0} c_{0}^{2}}\left\langle p_{1}^{2}\right\rangle-\frac{1}{2} \rho_{0}\left\langle\left|\mathbf{v}_{1}\right|^{2}\right\rangle,
$$

where $c_{0}$ is the sound propagation speed. The same goes for the ARF that can be expressed using an integral over the close surface $S$ delimiting a chosen volume ${ }^{13,15,16}$

$$
\mathbf{F}=\iint_{S}\left[-\frac{1}{2 \rho_{0} c_{0}^{2}}\left\langle p_{1}^{2}\right\rangle \mathbf{n}+\frac{1}{2} \rho_{0}\left\langle\left|\mathbf{v}_{1}\right|^{2}\right\rangle \mathbf{n}-\rho_{0}\left\langle\mathbf{v}_{1}\left(\mathbf{v}_{1} \cdot \mathbf{n}\right)\right\rangle\right] d S,
$$

where $\mathbf{n}$ is the vector normal to $S$ and pointing outwards.
The expression given in Eq. (11) is obtained for an inviscid fluid. An equivalent expression for a viscous fluid was obtained by Danilov and Mironov ${ }^{12}$ but its computation is more involved. In the rest of the document the 1 subscript is dropped for convenience and the quantities $p$ and $\mathbf{v}$ refer to the respective first order quantities associated with the acoustic perturbations instead of total quantities as before.

The ARF applied to an element of volume in $\mathrm{N} / \mathrm{m}^{3}$ is

$$
F_{i}=-\frac{\partial\left\langle p_{E}\right\rangle}{\partial x_{i}}-\rho_{0} \frac{\partial\left\langle v_{i} v_{k}\right\rangle}{\partial x_{k}} \text {. }
$$

Some words should be said about the validity of Eq. (11). It is strictly valid only for propagation in fluids where there can be no shearing and the shear modulus is null. Indeed, it assumes that the surface forces on each volume element are only due to the pressure. This approximation is reasonable in the case of ultrasound propagation in tissue where the bulk modulus (on the order of several GPa) is much greater than the shear modulus (several $\mathrm{kPa})^{17}$ and compressional stresses appear much more readily than shear stresses.

Although Eq. (11) is derived assuming the fluid is inviscid and homogeneous, it does account, albeit indirectly, for the wave momentum deposition in the volume inside the close surface $S$ due to viscous attenuation and possible scattering due to inhomogeneities. Strictly speaking, together with the inviscid stresses included in Eq. (11), viscous stresses should also be added. However, those stresses can become comparable to the inviscid stresses on the surface $S$ only at the interfaces between two different media or at a region of abrupt changes of the medium properties, i.e., in strongly inhomogeneous media, which are not considered here. In a practical setting for medical ultrasound applications, soft tissues are characterized by fairly low absorption and scattering, which justifies the use of the inviscid stress tensor in the integrand of Eq. (11).

\section{Quasi-plane wave approximation}

In general the mean Eulerian excess pressure $\left\langle p_{E}\right\rangle$ is not null however when the wave has the form of a bounded but wide quasi-plane wave this pressure can be approximated to zero. This follows from the fact that for a plane wave $p=\rho_{0} c_{0} v$ where $v$ is the projection of the particle velocity vector $\mathbf{v}$ on the propagation direction, i.e., $p_{E}=0$ according to Eq. (10). Thus for a quasi-plane wave $p_{E}$ can be considered as negligible. The expression for the ARF then becomes

$$
F_{i}=-\rho_{0} \frac{\partial\left\langle v_{i} v_{k}\right\rangle}{\partial x_{k}},
$$

or in the vector notation

$$
\mathbf{F}=-\rho_{0}\langle\mathbf{v}(\nabla \cdot \mathbf{v})+(\mathbf{v} \cdot \nabla) \mathbf{v}\rangle,
$$

which is what Nyborg derived in Ref. 14.

\section{Attenuated plane wave}

Further, if the wave is considered purely plane the ARF is directed in the wave propagation direction $x_{i}$ and using Eq. (13), 


$$
F=F_{i}=-\rho_{0} \frac{\partial\left\langle v^{2}\right\rangle}{\partial x_{i}}=-\frac{1}{c_{0}} \frac{\partial I}{\partial x_{i}},
$$

where $v=v_{i}$ is the particle velocity amplitude and $I$ $=\left\langle\rho_{0} c_{0} v^{2}\right\rangle$ is the acoustic intensity of the plane wave. If the wave is exponentially attenuated with an attenuation coefficient $\alpha$,

$$
\frac{\partial I}{\partial x_{i}}=-2 \alpha I
$$

and

$$
F=\frac{2 \alpha I}{c_{0}} .
$$

This expression is widely used when modeling the ARF used in elastography. $6,18,19$

Since the pressure is easier to measure or simulate than the particle velocity, in practice the expression

$$
I=\frac{\left\langle p^{2}\right\rangle}{\rho_{0} c_{0}}
$$

is often used for the wave intensity.

As shown in Sec. III, when using a three-dimensional derivation and the generic expression for the wave intensity $\mathbf{I}=\langle p \mathbf{v}\rangle$, Eq. (17) can be generalized to non-plane waves. In that case, the expression $\mathbf{F}=2 \alpha \mathbf{I} / c_{0}$ becomes an alternate formulation of the second-order approximation. It is therefore important to note that it is the expression for the wave intensity, Eq. (18), that translates the approximation of a plane wave rather than the expression for the ARF, Eq. (17).

\section{EXPRESSION OF THE ARF IN A VISCOUS FLUID}

In this section we derive an expression for the ARF using only the spatial derivatives of the pressure field. For clarity, we explicitly use the variables $x, y, z$ to refer to the ARF components and call them $F_{x}, F_{y}$, and $F_{z}$ in place of $F_{i}$ used in Sec. II. We present the detailed calculations for the $z$ component of the ARF only and the $x$ and $y$ components are deduced by appropriate variable replacement.

\section{A. Second-order approximation}

Using the second-order approximation formulation [Eq. (12)] the $z$ component of the ARF is

$$
\begin{aligned}
-F_{z}(x, y, z)= & \rho_{0}\left[\frac{\partial\left\langle v_{x} v_{z}\right\rangle}{\partial x}+\frac{\partial\left\langle v_{y} v_{z}\right\rangle}{\partial y}\right] \\
& +\frac{\partial}{\partial z}\left[\rho_{0} \frac{\left\langle v_{z}^{2}-v_{x}^{2}-v_{y}^{2}\right\rangle}{2}+\frac{\left\langle p^{2}\right\rangle}{2 \rho_{0} c_{0}^{2}}\right] \\
= & \rho_{0} \nabla \cdot\left\langle\mathbf{v} v_{z}\right\rangle \\
& +\frac{\partial}{\partial z}\left[-\rho_{0} \frac{\left\langle v_{x}^{2}+v_{y}^{2}+v_{z}^{2}\right\rangle}{2}+\frac{\left\langle p^{2}\right\rangle}{2 \rho_{0} c_{0}^{2}}\right],
\end{aligned}
$$

where $\mathbf{v}=\left(v_{x}, v_{y}, v_{z}\right)$.
Let us consider a harmonic wave of angular frequency $\omega$ and introduce the complex amplitudes of the particle velocity components and acoustic pressure

$$
\begin{aligned}
v_{i} & =\frac{V_{i}}{2} e^{-j \omega t}+\frac{V_{i}^{*}}{2} e^{j \omega t}, \\
p & =\frac{P}{2} e^{-j \omega t}+\frac{P^{*}}{2} e^{j \omega t},
\end{aligned}
$$

where $i=x, y$, or $z, j$ is the imaginary unit, and the asterisks denote complex conjugates. We get

$$
\begin{aligned}
\boldsymbol{\nabla} \cdot\left\langle\mathbf{v} v_{z}\right\rangle= & \frac{V_{z}}{4}\left(\frac{\partial V_{x}^{*}}{\partial x}+\frac{\partial V_{y}^{*}}{\partial y}+\frac{\partial V_{z}^{*}}{\partial z}\right)+\frac{V_{x}^{*}}{4} \frac{\partial V_{z}}{\partial x} \\
& +\frac{V_{y}^{*}}{4} \frac{\partial V_{z}}{\partial y}+\frac{V_{z}^{*}}{4} \frac{\partial V_{z}}{\partial z}+\text { c.c. }
\end{aligned}
$$

and

$$
\begin{aligned}
& \frac{\partial\left\langle v_{i}^{2}\right\rangle}{\partial z}=\frac{V_{i}}{2} \frac{\partial V_{i}^{*}}{\partial z}+\text { c.c., } \\
& \frac{\partial\left\langle p^{2}\right\rangle}{\partial z}=\frac{P}{2} \frac{\partial P^{*}}{\partial z}+\text { c.c. },
\end{aligned}
$$

where $i=x, y$, or $z$ and c.c. designates the complex conjugate of the preceding expression. Note that in this formula the repeated indices on the right-hand side are not summed over (no Einstein summation).

Replacing those in the expression of $F_{z}(x, y, z)$, we get

$$
\begin{aligned}
-F_{z}(x, y, z) & \\
= & \frac{\rho_{0}}{4}\left[V_{z}\left(\frac{\partial V_{x}^{*}}{\partial x}+\frac{\partial V_{y}^{*}}{\partial y}+\frac{\partial V_{z}^{*}}{\partial z}\right)+V_{x}^{*} \frac{\partial V_{z}}{\partial x}+V_{y}^{*} \frac{\partial V_{z}}{\partial y}\right] \\
& -\frac{\rho_{0}}{4}\left[V_{x} \frac{\partial V_{x}^{*}}{\partial z}+V_{y} \frac{\partial V_{y}^{*}}{\partial z}\right]+\frac{1}{4 \rho_{0} c_{0}^{2}} P \frac{\partial P^{*}}{\partial z}+\text { c.c. }(20)
\end{aligned}
$$

Combining the linearized continuity equation and equation of state

$$
\begin{aligned}
& \frac{\partial \rho}{\partial t}+\rho_{0} \boldsymbol{\nabla} \cdot \mathbf{v}=0, \\
& p=c_{0}^{2} \rho
\end{aligned}
$$

we get the relation for the complex amplitudes

$$
\frac{j \omega}{\rho_{0} c_{0}^{2}} P=\frac{\partial V_{x}}{\partial x}+\frac{\partial V_{y}}{\partial y}+\frac{\partial V_{z}}{\partial z} .
$$

We can now replace $\partial V_{x}^{*} / \partial x+\partial V_{y}^{*} / \partial y+\partial V_{z}^{*} / \partial z$ in Eq. (20) to get

$$
\begin{aligned}
-F_{z}(x, y, z)= & \frac{\rho_{0}}{4}\left[-V_{z} \frac{j \omega}{\rho_{0} c_{0}^{2}} P^{*}+V_{x}^{*} \frac{\partial V_{z}}{\partial x}+V_{y}^{*} \frac{\partial V_{z}}{\partial y}\right. \\
& \left.-V_{x} \frac{\partial V_{x}^{*}}{\partial z}-V_{y} \frac{\partial V_{y}^{*}}{\partial z}\right]+\frac{1}{4 \rho_{0} c_{0}^{2}} P \frac{\partial P^{*}}{\partial z}+\text { c.c. }
\end{aligned}
$$

Let us now turn to the linearized equation of motion in a viscous fluid for an irrotational flow 


$$
\rho_{0} \frac{\partial \mathbf{v}}{\partial t}=-\nabla p+\left(\zeta+\frac{4}{3} \eta\right) \nabla(\nabla \cdot \mathbf{v})
$$

where $\zeta$ and $\eta$ are the bulk and shear viscosity, respectively.

Using Eqs. (21) and (22) to replace the divergence of $\mathbf{v}$ by the time derivative of $p$ in the previous equation we get the following expression for the complex amplitudes

$$
V_{x_{i}}=\left(\frac{1}{\rho_{0} j \omega}-\frac{\zeta+\frac{4}{3} \eta}{\rho_{0}^{2} c_{0}^{2}}\right) \frac{\partial P}{\partial x_{i}}
$$

where $x_{i}=x, y$, or $z$.

Using Eq. (25) in Eq. (24) we can see that all the terms in the square bracket except for the first one cancel each other out and we get

$$
-F_{z}(x, y, z)=\frac{\left(\zeta+\frac{4}{3} \eta\right) j \omega}{4 \rho_{0}^{2} c_{0}^{4}} \frac{\partial P}{\partial z} P^{*}+\text { c.c. }
$$

Introducing the attenuation coefficient $\alpha(\omega)=\left(\zeta+\frac{4}{3} \eta\right) \omega^{2} /$ $\left(2 \rho_{0} c_{0}^{3}\right)$ and extending for all three components of the ARF we can write in vector notation

$$
\mathbf{F}(x, y, z)=\frac{j \alpha(\omega)}{2 \omega \rho_{0} c_{0}}\left(P \nabla P^{*}-P^{*} \nabla P\right) .
$$

Note that according to Eq. (25), this equation can also be written in the following form:

$$
\mathbf{F}(x, y, z)=\frac{\alpha(\omega)}{2 c_{0}}\left(\mathbf{V}^{*} P+\text { c.c. }\right)+O\left(\alpha^{2}(\omega)\right) .
$$

Here $O\left(\alpha^{2}(\omega)\right)$ indicates the terms that are proportional to the square of the attenuation coefficient and that can be considered negligible in our case of a weakly absorbing media.

Also the wave intensity in such a weakly absorbing viscous media is $\mathbf{I}=\langle p \mathbf{v}\rangle=P \mathbf{V}^{*} / 4+$ c.c., so we come to a short expression of the ARF in the second-order approximation

$$
\mathbf{F}(x, y, z)=2 \alpha(\omega) \frac{\mathbf{I}}{c_{0}} .
$$

This shows that the expression $\mathbf{F}=2 \alpha(\omega) \mathbf{I} / c_{0}$ when using the definition of $\mathbf{I}$ in the generic case is an equivalent formulation of the ARF in the second-order approximation.

\section{B. Quasi-plane wave approximation}

According to Eqs. (12) and (13) the ARF in the quasiplane wave approximation can be obtained by adding the gradient of the mean Eulerian excess pressure to the expression of the ARF obtained by the second-order approximation. From Eq. (27) we have therefore

$$
\mathbf{F}=\frac{j \alpha(\omega)}{2 \omega \rho_{0} c_{0}}\left(P \nabla P^{*}-P^{*} \nabla P\right)+\nabla\left\langle p_{E}\right\rangle,
$$

for the quasi-plane wave approximation.
Using Eqs. (10) and (25) we can express the mean Eulerian excess pressure as

$$
\left\langle p_{E}\right\rangle=\frac{P P^{*}}{4 \rho_{0} c_{0}^{2}}-\frac{\rho_{0}}{4} A A^{*} \nabla P \nabla P^{*}
$$

where

$$
A=\frac{1}{j \rho_{0} \omega}-\frac{\zeta+\frac{4}{3} \eta}{\rho_{0}^{2} c_{0}^{2}}=\frac{1}{j \rho_{0} \omega}-\frac{2 \alpha(\omega) c_{0}}{\rho_{0} \omega^{2}} .
$$

The expression for the ARF becomes

$$
\mathbf{F}=\frac{j \omega A}{4 c_{0}^{2}} P^{*} \nabla P-\frac{\rho_{0} A A^{*}}{4} \nabla^{2} P \nabla P^{*}+\text { c.c. }
$$

\section{Attenuated plane wave approximation}

In the case of a plane wave $p=\rho_{0} c_{0} v$ and we have

$$
F=2 \alpha(\omega) \frac{I}{c_{0}}=\alpha(\omega) \frac{P^{2}}{\rho_{0} c_{0}^{2}} .
$$

In the rest of the article the attenuated plane wave approximation uses $I=P^{2} / \rho_{0} c_{0}$ for the intensity and Eq. (33) for the ARF.

Note that when this approximation is used in typical cases such as a focused diagnostic transducer where the pressure field differs from a plane wave, the result is a scalar and does not provide any information about the ARF direction. Some assumptions must therefore be made about the direction of the force in this case. This is explained in more detail in Sec. IV.

\section{ANALYTICAL EXPRESSION OF THE ARF FOR PARTICULAR CASES}

In this section we consider two particular cases for which an analytical expression of the ARF can be found. The first case considered is that of a spherical concave source with uniform distribution normal velocity. In this case, we can derive an expression for the ARF along the transducer axis. The second case is that of a quasi-Gaussian beam satisfying the Helmholtz equation. ${ }^{20}$

\section{A. Spherical concave source}

We consider a spherical concave source with radius of curvature $R$, half opening angle $\theta$, diameter $D$, and depth $h$ (Fig. 1). The normal velocity at the face of the source is considered constant and equal to $V_{0}$. Using the Rayleigh integral O'Neil established the following expression for the complex pressure amplitude on the transducer main axis: ${ }^{21}$

$$
P(0,0, z)=\rho_{0} c_{0} V_{0} \frac{e^{j k z}-e^{j k R_{\max }}}{1-\frac{z}{R}},
$$

where $z$ is the distance to the transducer center and $R_{\max }$ is the distance from the observation point to the transducer edge (Fig. 1) 


$$
R_{\max }=R \sqrt{1+\left(1-\frac{z}{R}\right)^{2}-2\left(1-\frac{z}{R}\right) \cos \theta} .
$$

Equation (34) is valid when $D$ and $h$ are large compared to the wavelength $\lambda$.

Using this equation and the expressions of the ARF established in Secs. III A and III B we want to establish an analytical expression for the ARF on the transducer axis. Due to symmetry, it is directed along the $z$ axis.

Detailed calculations that can be found in Appendix A give the expression for the ARF on the axis using the second-order approximation

$$
F_{z}(0,0, z)=\frac{\alpha(\omega) P_{0}^{2}}{\rho_{0} c_{0}^{2}} \frac{\left(R_{\max }^{\prime}+1\right)}{\left(1-\frac{z}{R}\right)^{2}}\left[1-\cos \left(\frac{\omega}{c_{0}}\left(z-R_{\max }\right)\right)\right],
$$

where

$$
\begin{aligned}
& R_{\max }^{\prime}=\frac{\partial R_{\max }}{\partial z}=\frac{\frac{z}{R}-1+\cos \theta}{\sqrt{1+\left(1-\frac{z}{R}\right)^{2}-2\left(1-\frac{z}{R}\right) \cos \theta}}, \\
& P_{0}=\rho_{0} c_{0} V_{0} .
\end{aligned}
$$

In the quasi-plane wave approximation due to symmetry the velocity vector and the pressure gradient are oriented along the $z$ axis and

$$
F_{z}(0,0, z)=\frac{j \omega A}{4 c_{0}^{2}} P^{*} \frac{\partial P}{\partial z}-\frac{\rho_{0} A A^{*}}{4} \frac{\partial^{2} P}{\partial z^{2}} \frac{\partial P^{*}}{\partial z}+\text { c.c. }
$$

The details derivation as well as the expressions for $\partial P / \partial z$ and $\partial^{2} P / \partial z^{2}$ can be found in Appendixes A and B.

We now use the above expressions to compare the amplitude of the ARF using the second-order approximation,

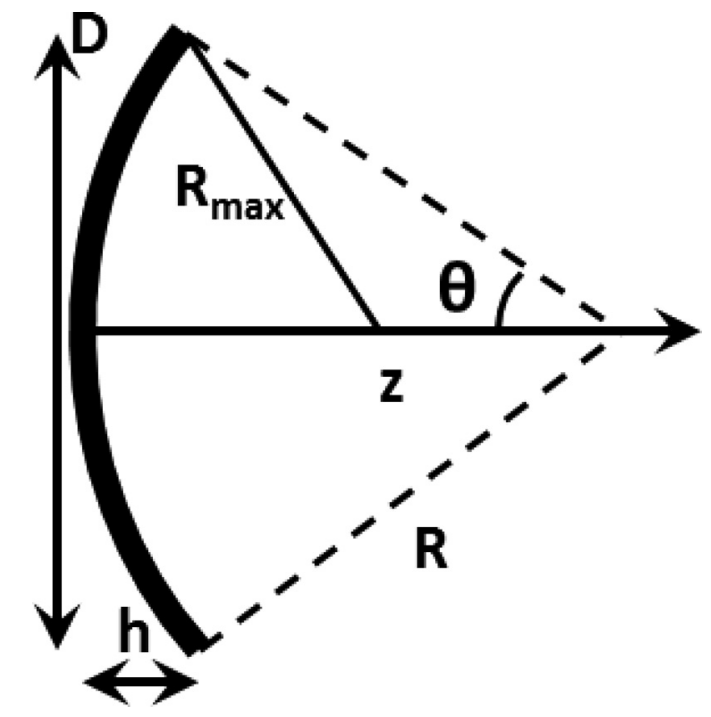

FIG. 1. Concave spherical source with radius of curvature $R$, diameter $D$, depth $h$, and half opening angle $\theta . R_{\max }$ is the distance between the observation point $(0,0, z)$ along the axis and the transducer edge. the quasi-plane wave approximation, and the attenuated plane wave approximation along the source axis for different values of the angle $\theta$ or equivalently different values of $F$ number which in our case is equal to $1 /(2 \sin \theta)$. A large value of $\theta$ corresponds to a highly focused transducer and a low $F$-number.

Figure 2 shows a comparison of the ARF amplitude for three configurations: $\theta=15^{\circ}, \theta=20^{\circ}, \theta=30^{\circ}$, and $\theta=45^{\circ}$ ( $F$-number of 1.9, 1.5, 1.0, and 0.7). The values chosen for $R$, $\rho_{0}$, and $c_{0}$ are $10 \mathrm{~cm}, 1000 \mathrm{~kg} / \mathrm{m}^{3}$, and $1500 \mathrm{~m} / \mathrm{s}$, respectively. The wave frequency is $3 \mathrm{MHz}$, the wavelength $\lambda=0.5 \mathrm{~mm}$, and the attenuation coefficient is $1 \mathrm{~dB} / \mathrm{cm} / \mathrm{MHz}^{2}$ Each configuration corresponds to a diameter $D$ of 5.2, 6.8, 10.0, and $14.1 \mathrm{~cm}$ and to a depth of 3.4, 6.0, 13.4, and $29.3 \mathrm{~mm}$.

As the source angle $\theta$ increases (the $F$-number decreases) the discrepancy at focus between results obtained with the second-order approximation and those obtained with the quasi-plane wave or the plane wave approximation increases. To quantify this discrepancy we compute $\Delta_{p w}$ and $\Delta_{q p}$ the root-mean-square errors (RMSEs) over the displayed depth range $(0.9 \leq z / R \leq 1.1)$ between the plane wave and the second-order approximations and between the quasiplane wave and the second-order approximations, respectively. The RMSE gives a relative measurement of the discrepancies between each solution. While $\Delta_{p w}$ and $\Delta_{q p}$ are very low for $F$-numbers greater than 1 they increase dramatically for $F$-number below 1 (Fig. 2 ).

This behaviour is expected since the pressure field distribution deviates greatly from a "locally plane wave" as the degree of focusing increases (increasing value of $\theta$ ).

These results clearly show that in the case of highly focused sources the quasi-plane wave and plane wave approximations significantly differ from the original expression of the second-order approximation.

\section{B. Quasi-Gaussian beam}

Quasi-Gaussian beams satisfying the Helmholtz equation can be defined in various ways. ${ }^{22,23}$ In this article we use the formulation from Ref. 20 where Sapozhnikov defines an axisymmetric beam with a "quasi-Gaussian" pressure distribution along the radial dimension. This beam satisfies the Helmholtz equation and can be approximated by a Gaussian beam at short distances from the symmetry axis. It can be seen as the superposition of two sources and sinks with complex coordinates. The analytical expression for the pressure field complex amplitude in this beam is

$$
\begin{aligned}
P(x, y, z)= & \frac{P_{0} z_{d}}{2 \sinh ^{2}\left(k z_{d}\right)}\left[e^{k z_{d}} \frac{\sin \left(k \sqrt{D_{-}}\right)}{\sqrt{D_{-}}}\right. \\
& \left.-e^{-k z_{d}} \frac{\sin \left(k \sqrt{D_{+}}\right)}{\sqrt{D_{+}}}\right]
\end{aligned}
$$

where

$$
\begin{aligned}
& z_{d}=k a^{2} / 2, \\
& D_{-}=x^{2}+y^{2}+\left(z-j z_{d}\right)^{2}, \\
& D_{+}=x^{2}+y^{2}+\left(z+j z_{d}\right)^{2},
\end{aligned}
$$



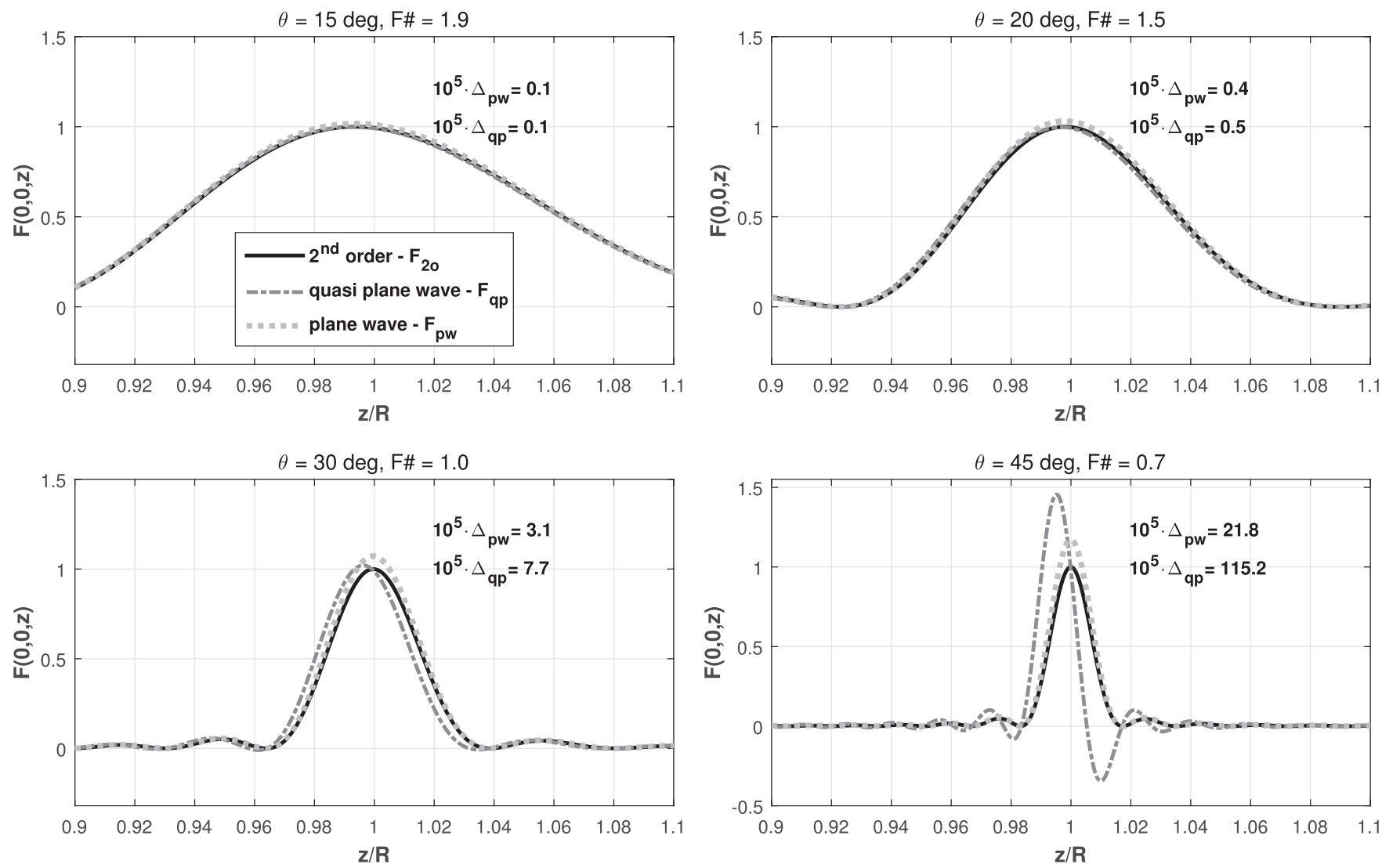

FIG. 2. Amplitude of the ARF along the source axis for a spherical concave source when using the second-order- $F_{2 o}$ (solid line), the quasi-plane wave (dashed-dotted line) $-F_{q p}$, and the plane wave $-F_{p w}$ (dotted line) approximations. Calculations were done for four setups with increasing values of $\theta$ (increasing degree of focusing). The ARF amplitude is normalized by the maximum of the amplitude found using the second-order approximation. $\Delta_{p w}$ and $\Delta_{q p}$ are the RMSEs over the displayed depth range between the plane wave and the second-order approximations and between the quasi-plane wave and the secondorder approximations, respectively.

with $a$ a parameter characteristic of the source spatial extension in the plane $z=0$.

The pressure field of such a beam in a plane containing the symmetry axis is shown in Fig. 3 for $k a=5$. It presents a maximum on the axis at $z=0$. The wavefield propagates from $z<0$ toward $z>0$ and presents a beam waist at $z=0$.

From the expression in Eq. (39) $P$ and its spatial derivatives can be computed anywhere and hence an analytical expression for ARF using the second-order or the quasiplane wave approximation can be established.

The detailed analytical expressions for the spatial derivatives or the pressure are given in Appendix C.

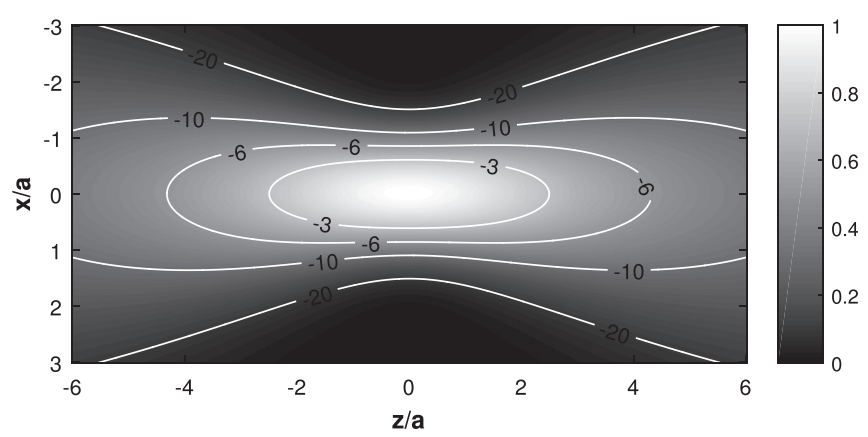

FIG. 3. Normalized pressure amplitude generated by a quasi-Gaussian beam in a plane containing the symmetry axis for $k a=5$. The wavefield propagates from $z<0$ toward $z>0$ and has a beam waist at $z=0$. The contours show the normalized level in $\mathrm{dB}$.
Using these expressions, we compute the ARF direction and amplitude in the plane $y=0$ using the second-order and quasi-plane wave approximations for $k a=7$ and $k a=5$. The higher the value of $k a$ the more "collimated" the beam is. The values for $\rho_{0}$ and $c_{0}$ are specified in Sec. IV A. The attenuation and frequency are as before $1 \mathrm{~dB} / \mathrm{cm} / \mathrm{MHz}^{2}$ and $3 \mathrm{MHz}$, respectively.

Figures 4 and 5 show the ARF field and amplitude using both formulations as well as the force amplitude on axis for all three formulations in the case $k a=7$ and $k a=5$, respectively. The on-axis force amplitudes are normalized with the maximum amplitude obtained using the second-order approximation. The quasi-plane wave approximation differs significantly from the results given using the second-order approximation especially for the low value of $k a$ where the beam is more divergent. The attenuated plane wave formulation over-estimates the force amplitude in $z=0$ compared to that obtained with the second-order approximation by $4 \%$ and $9 \%$ for $k a=7$ and $k a=5$, respectively.

This shows that in this case the second-order approximation should be favoured especially for low values of $k a$.

\section{INFLUENCE OF THE MEAN EULERIAN EXCESS PRESSURE}

The expression for the ARF in the quasi-plane wave approximation is obtained by adding the mean Eulerian 

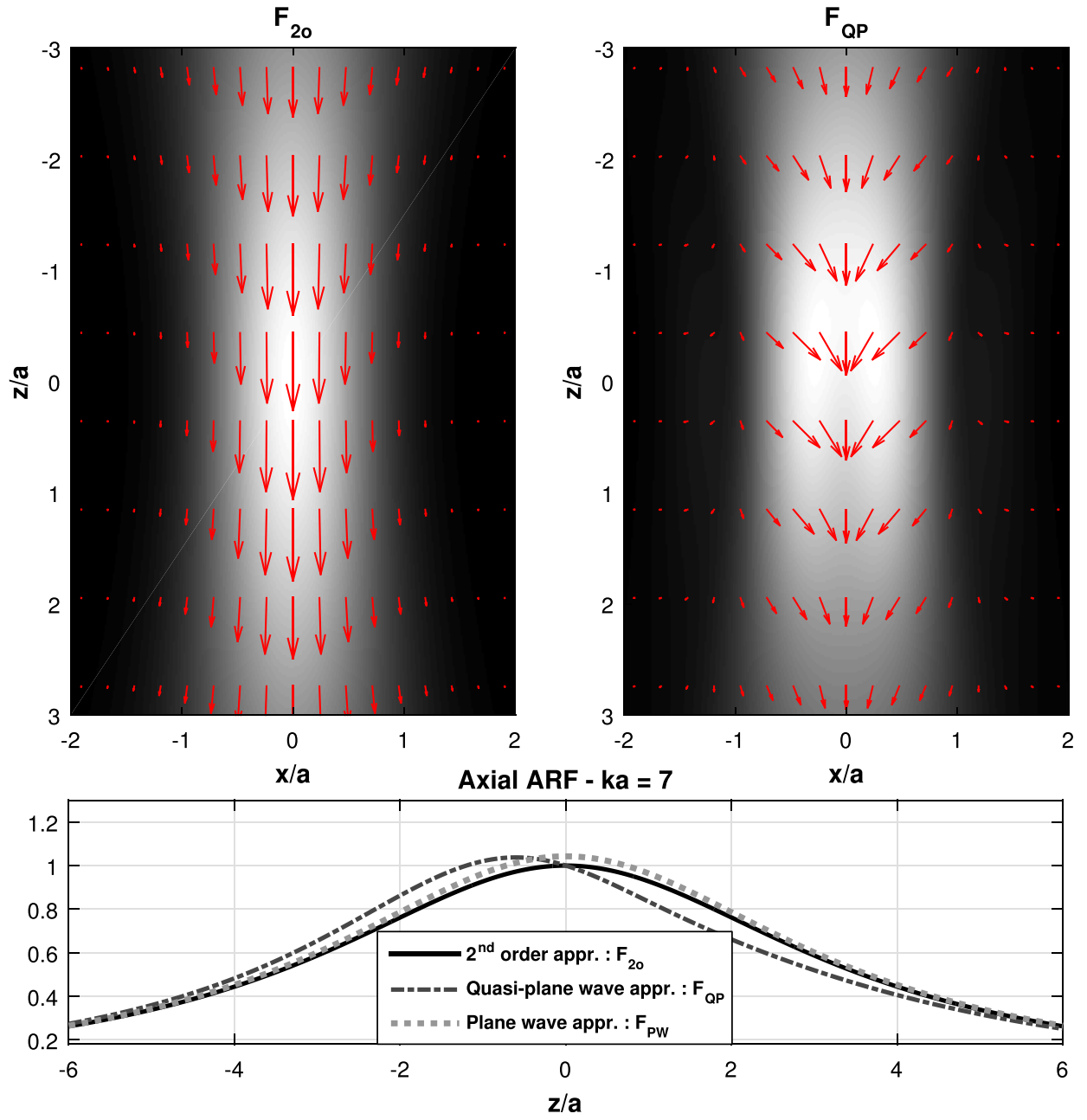

FIG. 4. (Color online) Amplitude of the ARF computed using the second-order approximation (top left) and the quasiplane wave approximation (top right) in the case $k a=7$. The gray scale represents the amplitude of the ARF and the arrows its direction. The ARF amplitude on the source symmetry axis $(x=0)$ is also compared (bottom) using the second-order approximation (solid line), the quasi-plane wave approximation (dashed-dotted line), and the plane wave approximation (dotted line). The on-axis force amplitudes are normalized with the maximum amplitude obtained using the second-order approximation. excess pressure $\left\langle p_{E}\right\rangle$ to the ARF expression obtained with the second-order approximation [see Eqs. (12) and (13)]. Considering Eqs. (30) and (31) it appears clearly that even in a lossless medium where $\alpha(\omega)=0$, the ARF in the quasiplane wave approximation is not null. This result is not physical. It is not the case for the ARF expressed using the second-order or quasi-plane wave approximation where it is proportional to $\alpha(\omega)$ in both cases.

Figure 6 shows the on-axis ARF in the lossless case using the quasi-plane wave approximation for a spherical concave source of half opening angle $\theta=20^{\circ}$ and for a quasi-Gaussian beam with $k a=7$. In the lossless case it corresponds to $\nabla\left\langle p_{E}\right\rangle$. It shows that the influence of the Eulerian pressure is mainly on each side of the focus depth and of the beam waist for the spherical concave source and the quasiGaussian beam, respectively.

It is therefore important to take the mean Eulerian excess pressure to correctly estimate the ARF.

However, as explained in Refs. 24 and 25 the component of the ARF that can be expressed as a gradient does not generate rotational motion in the medium that is associated with shear waves in case of soft solids, and with acoustic streaming in case of fluids. So although $\left\langle p_{E}\right\rangle$ should be taken into account for a correct computation of the ARF, it does not contribute to the generation of a displacement of shear waves.
In the context of SWEI the gradient of the mean Eulerian excess pressure can therefore be neglected to estimate the displacement created by the ARF and the secondorder and quasi-plane wave approximations should lead to identical displacements.

\section{NUMERICAL SIMULATION OF THE ARF FROM AN EXISTING ULTRASOUND SOURCE}

The analytical expressions presented in Sec. IV correspond to somewhat idealistic sources. In practice, sources are of a more complex structure. For instance, in SWEI the probes are multi-element arrays similar or identical to those used in conventional ultrasound imaging. In such a case, analytical expressions for the ARF do not exist, but the acoustic field and related radiation force can be calculated based on direct numerical simulations. To do so, in this paper we used the simulation package $\mathrm{k}-\mathrm{Wave}^{26,27}$ running under MATLAB (Mathworks, Natick, MA).

We simulated the pressure and particle velocity fields created by an ultrasound transducer P4-2 V (Verasonics, Inc., Redmond, WA). This transducer is a $20-\mathrm{mm}$ wide and 14-mm high phased array made of 64 elements. It has a center frequency around $3 \mathrm{MHz}$, a fixed focus in the elevation direction at $60 \mathrm{~mm}$, and an adjustable electronic focus in the azimuth direction set to $30 \mathrm{~mm}$ in our simulations. 


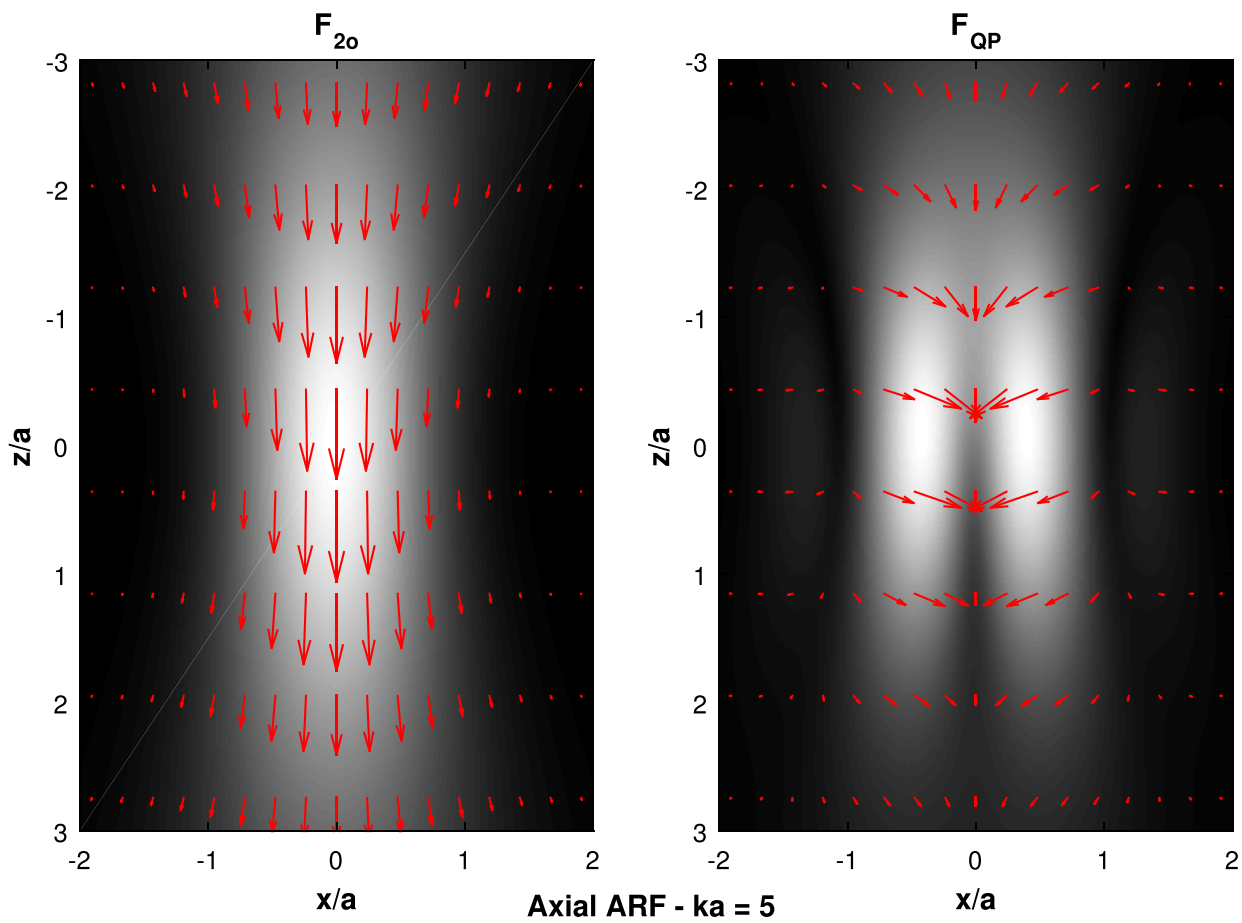

FIG. 5. (Color online) Amplitude of the ARF computed using the secondorder approximation (top left) and the quasi-plane wave approximation (top right) in the case $k a=5$. The gray scale represents the amplitude of the ARF and the arrows its direction. The ARF amplitude on the source symmetry axis $(x=0)$ is also compared (bottom) using the second-order approximation (solid line), the quasi-plane wave approximation (dashed-dotted line), and the plane wave approximation (dotted line). The on-axis force amplitudes are normalized with the maximum amplitude obtained using the second-order approximation.

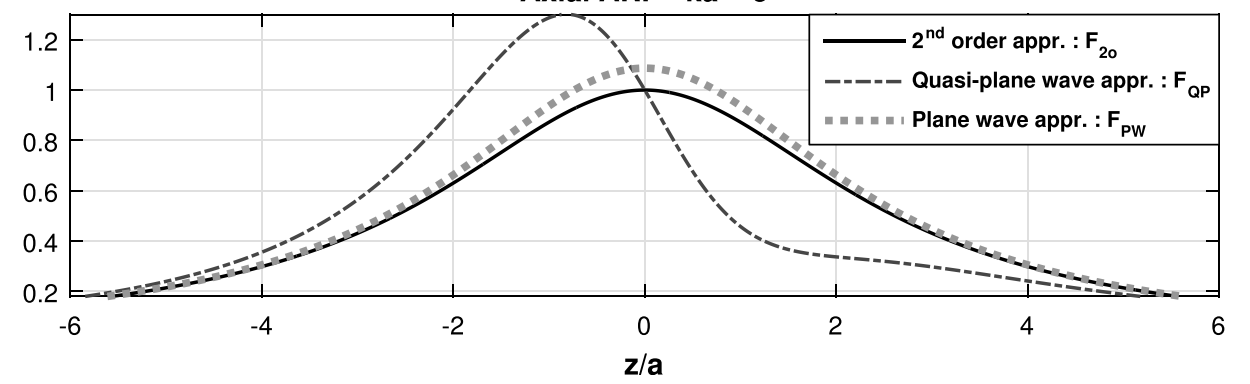

The propagation medium is chosen homogeneous with a sound speed and density of $1540 \mathrm{~m} / \mathrm{s}$ and $980 \mathrm{~kg} / \mathrm{m}^{3}$, respectively. The attenuation coefficient is set to $0.5 \mathrm{~dB} / \mathrm{MHz} / \mathrm{cm}$. The transmitted pulse is 5-cycles long and is obtained by weighting a $3-\mathrm{MHz}$ sine wave by a Gaussian envelope. Waveform distortion effects due to acoustic nonlinearity are not included.

The computation grid is made of 133, 93, and 321 elements in the azimuth, elevation, and depth direction, respectively. The mesh size is set to $0.16 \mathrm{~mm}$ corresponding to a total grid size of approximately $21.3 \mathrm{~mm}$ in azimuth, $14.9 \mathrm{~mm}$ in elevation, and $51.4 \mathrm{~mm}$ in depth. The simulation end time is set to $40 \mu \mathrm{s}$ and the time step is $0.03 \mu \mathrm{s}$. A perfectly matched layer (PML) 20-element wide in the azimuth and depth directions and 10-element wide in the elevation direction is included in the computation grid.

The simulations were run in three dimensions but the ARF was only computed in the plane of symmetry for the
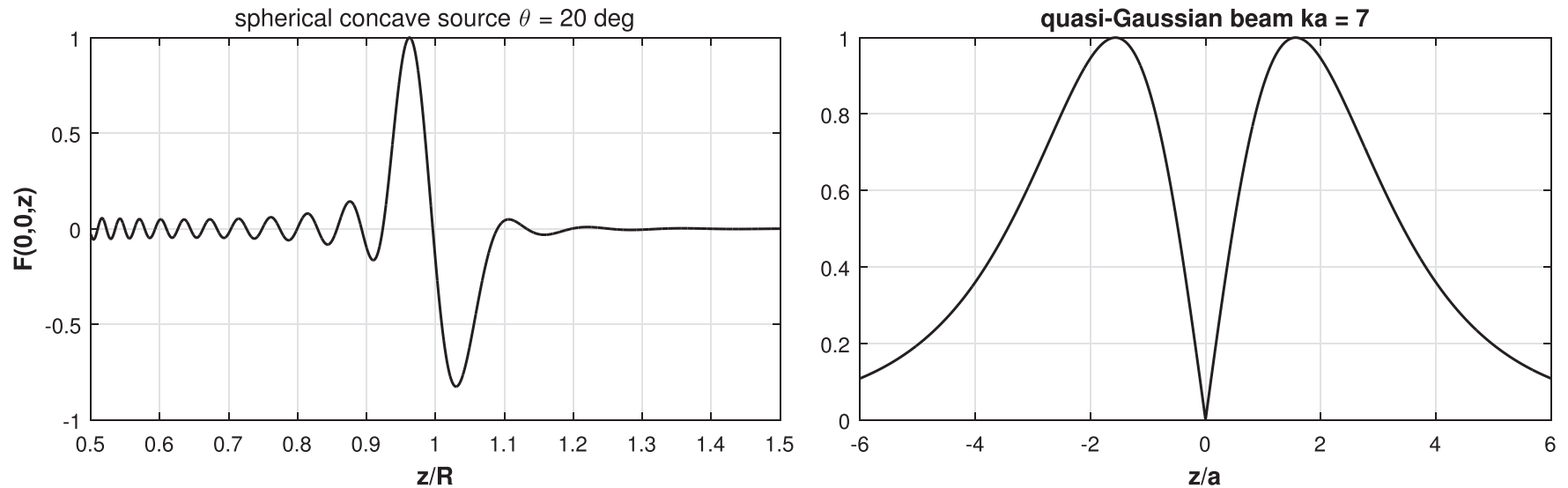

FIG. 6. Normalized ARF on the axis of a spherical concave source of half opening angle $\theta=20^{\circ}$ (left) and on the axis of a quasi-Gaussian beam with $k a=7$ (right) using the quasi-plane wave approximation in the lossless case. In that case the ARF is equal to $\nabla\left\langle p_{E}\right\rangle$. 


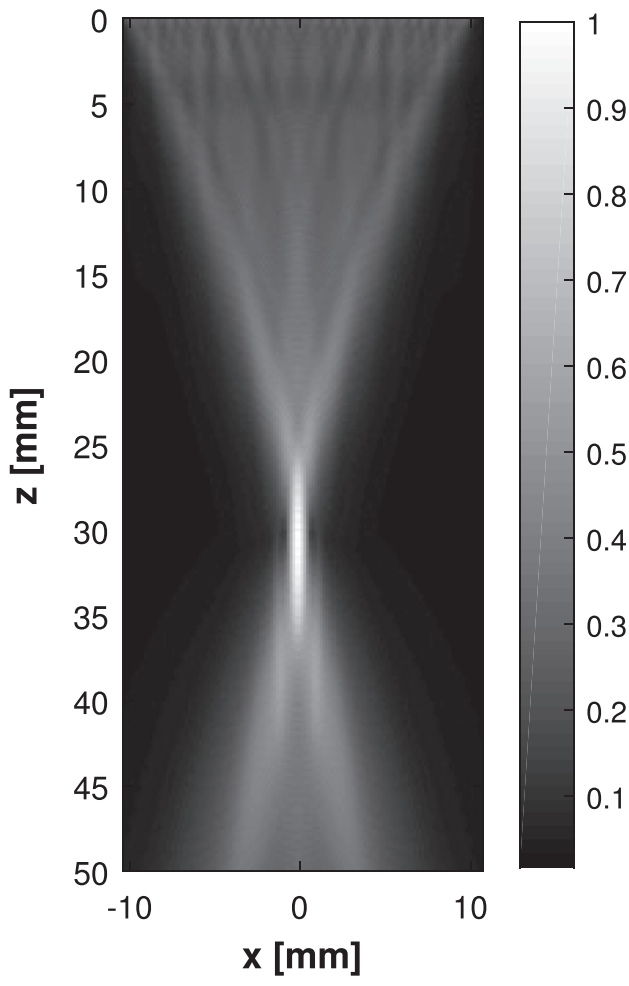

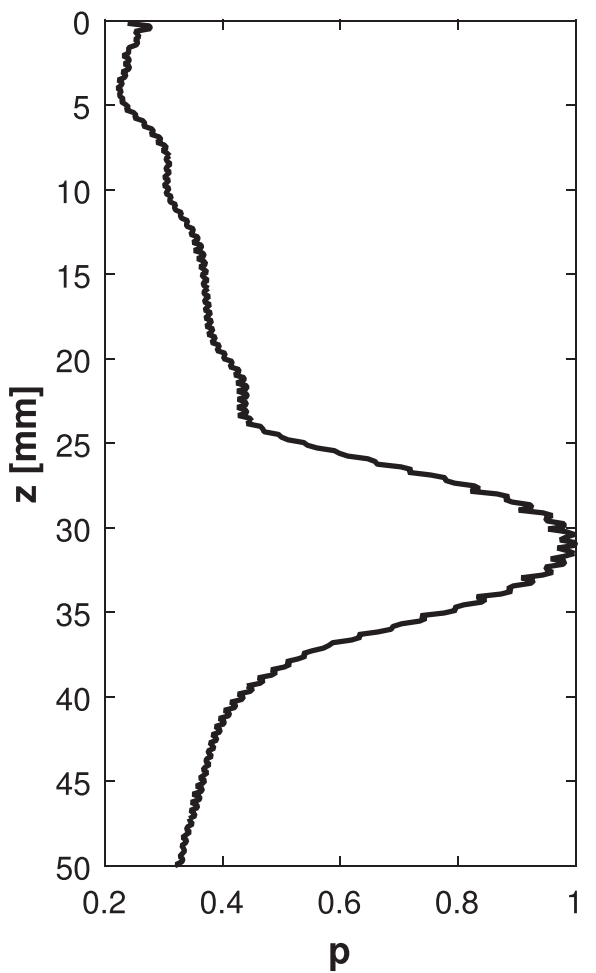

FIG. 7. Spatial distribution of the normalized pressure amplitude in the $x, z$ plane (left) and along the transducer main propagation axis, $x=0$ (right) for a propagation in a homogeneous medium. transducer (zero elevation). In this plane, hereafter called $x$, $z$ plane, the ARF has only two components $F_{x}$ and $F_{z}$ due to symmetry.

The pressure amplitude distribution obtained is shown in Fig. 7.

The distribution of the ARF formulated using Eq. (12) is shown in the left pane of Fig. 8.

It shows that the ARF reaches a maximum amplitude along the main propagation axis in the focal area.

When using Eq. (13) we obtain the spatial distribution of the ARF shown in the middle pane of Fig. 8.
This spatial distribution is quite similar to that obtained with the ARF formulation using the second-order approximation. The main difference is in the distribution of $F_{x}$.

Finally, Eqs. (17) and (18) valid for an attenuated plane wave were used to get the spatial distribution of the ARF shown in the right pane of Fig. 8.

As the force has only one component in this case (along the plane wave propagation direction) a common approximation sets the force direction toward the focal point for depths smaller than focus and away from the focal point for depths larger than focus (Poynting vector). In a reduced depth range
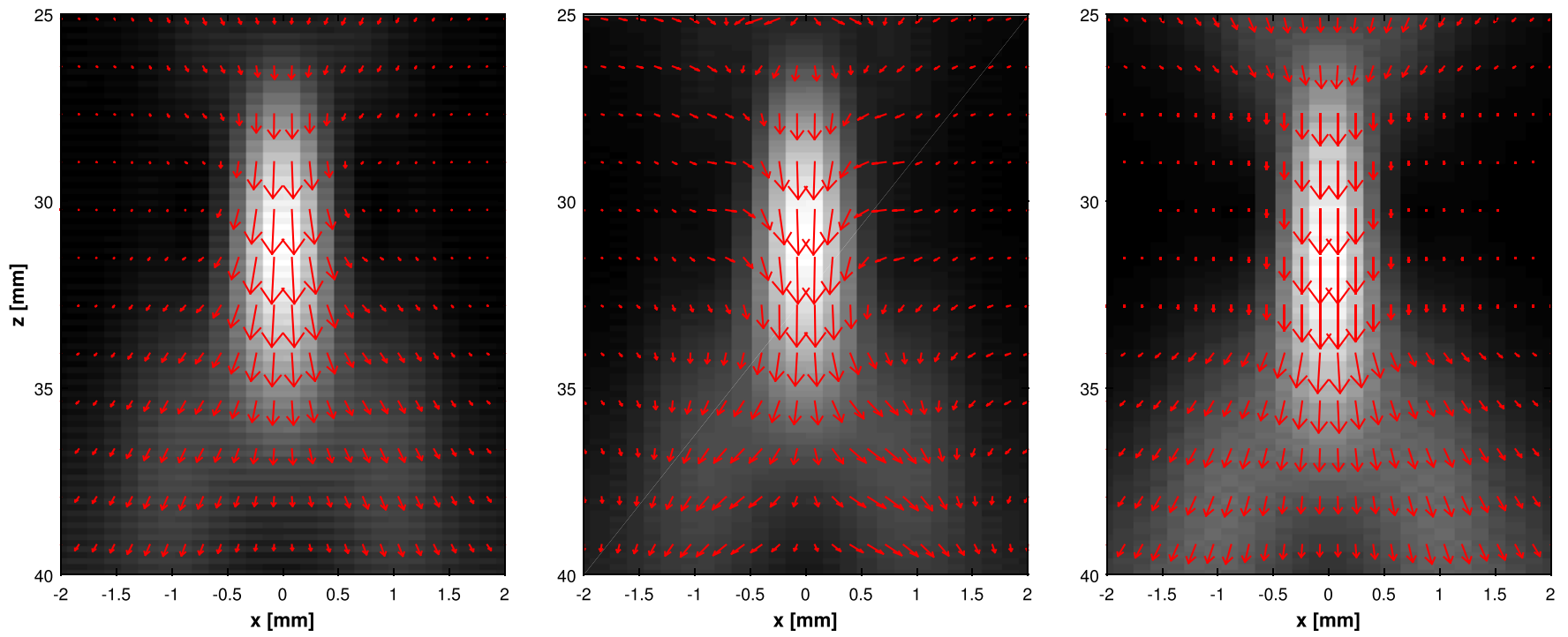

FIG. 8. (Color online) Spatial distribution of the ARF in the zero-elevation plane when using the second-order approximation (left), the quasi-plane wave approximation (middle), and the attenuated plane wave approximation (right). The gray tones represent the normalized ARF amplitude. The length and direction of the arrows represent the local amplitude and direction for the ARF. Note that the vertical and horizontal scales are not identical for better readability. 
around focus, typically $10 \%$ of the focus distance, the force is approximated parallel to the main propagation axis. ${ }^{6}$ This approximation was also made in our case.

\section{SIMULATION OF SHEAR WAVE PROPAGATION}

Once the spatial distribution of the ARF is available the propagation of the ensuing shear wave can be modelled. For that, we used k-Wave again to simulate propagation of elastic waves in three dimensions. ${ }^{28,29}$

The ARF is considered as an input volume force distributed in the $x, z$ plane $(y=0)$. The ARF amplitude is taken constant in time and it is applied for a given duration. In our simulations, the force is applied at $t=0$ for $200 \mu$ s which corresponds to a 600-period long pushing pulse of center frequency $3 \mathrm{MHz}$.

The medium is homogeneous with a shear wave speed of $1.7 \mathrm{~m} / \mathrm{s}$. The computation grid is made of 128 elements in the azimuth and depth directions and 32 elements in the elevation direction. The mesh size is set to $0.2 \mathrm{~mm}$ corresponding to a total grid size of $25.6 \mathrm{~mm}$ in the azimuth and depth directions and $6.4 \mathrm{~mm}$ in the elevation direction. The simulation end time is set to $5.5 \mathrm{~ms}$ with a time step of $1 \mu \mathrm{s}$. The PML size is 10 elements in the azimuth and depth directions and 5 elements in the elevation direction. Note that in $\mathrm{k}-$ Wave there is no formal way to input a volume force. It only takes stress components or particle velocities as inputs. However, as explained in the k-Wave user manual ${ }^{30}$ the input velocity is automatically scaled to a force per mass $\left(\mathrm{m} / \mathrm{s}^{2}\right)$ by multiplying it with the factor $2 c_{0} / \Delta x_{i}$ where $\Delta x_{i}$ is the spatial step in the chosen direction. To get a volume force $F_{i}$ as input, we can therefore use an input velocity equal to $\left(F_{i} / \rho_{0}\right) /\left(2 c_{0} / \Delta x_{i}\right)$.

The generated shear waves propagate mainly in the $x$ direction and the displacement main component is therefore along the $z$ direction. In SWEI and more generally in ultrasound-based elastography, only the z-component of the displacement is tracked (toward and away from the transducer). Therefore, we only study the displacement along $z$.

Using the fields of ARF computed with the three methods described above, the obtained displacements along the $z$ direction are shown in Fig. 9 at three distinct times.

Three cuts along the $x$ direction in each of these planes are shown in Fig. 10.

The displacements computed using the attenuated plane wave approximation for the ARF clearly differ from the two other computed displacements.
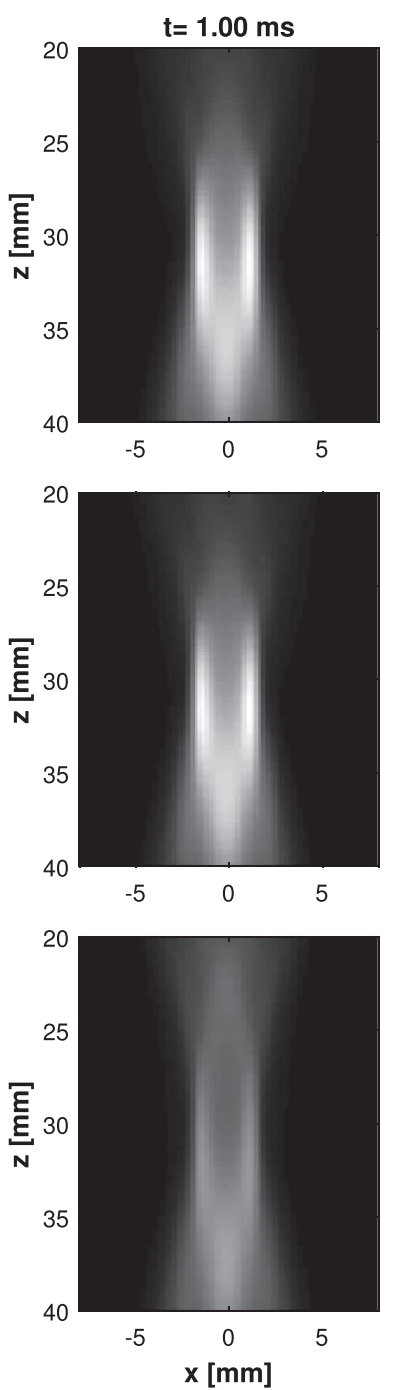
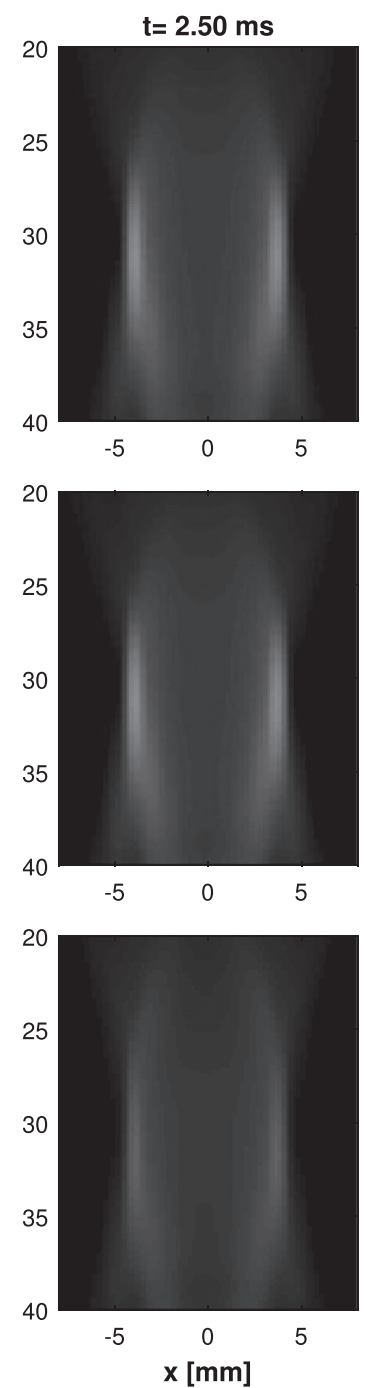
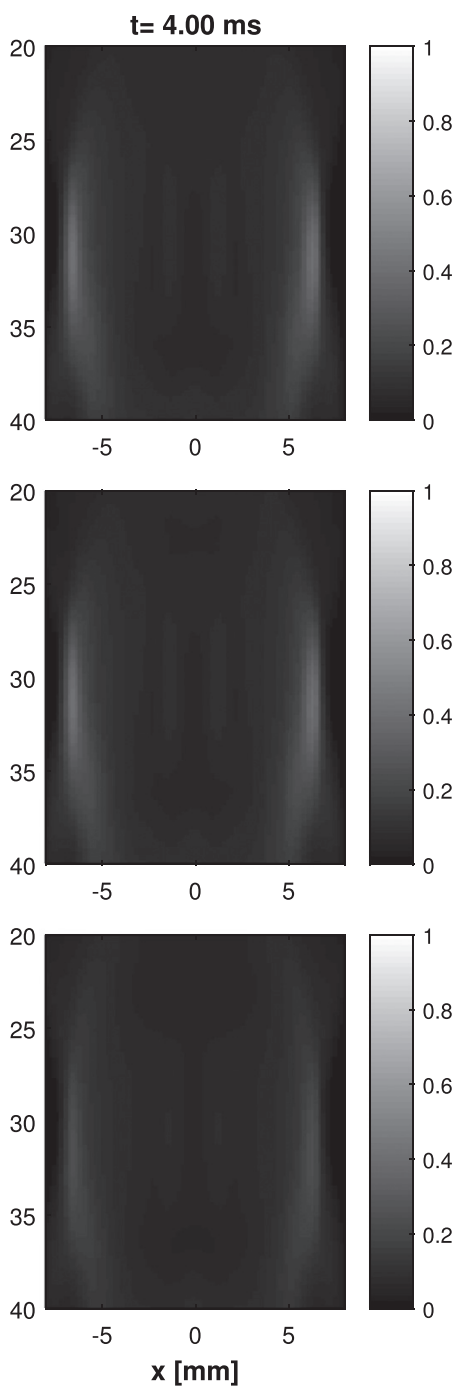

FIG. 9. Fields of displacement in a homogeneous medium at $t=1 \mathrm{~ms}$ (left column), $t=2.5 \mathrm{~ms}$ (middle column), and $t=4 \mathrm{~ms}$ (right column) generated by the ARF using the second-order approximation (top row), the quasiplane wave approximation (middle row), and the attenuated plane wave approximation (bottom row). The fields are normalized with the maximum displacements obtained from the second-order approximation at $t=1 \mathrm{~ms}$. 

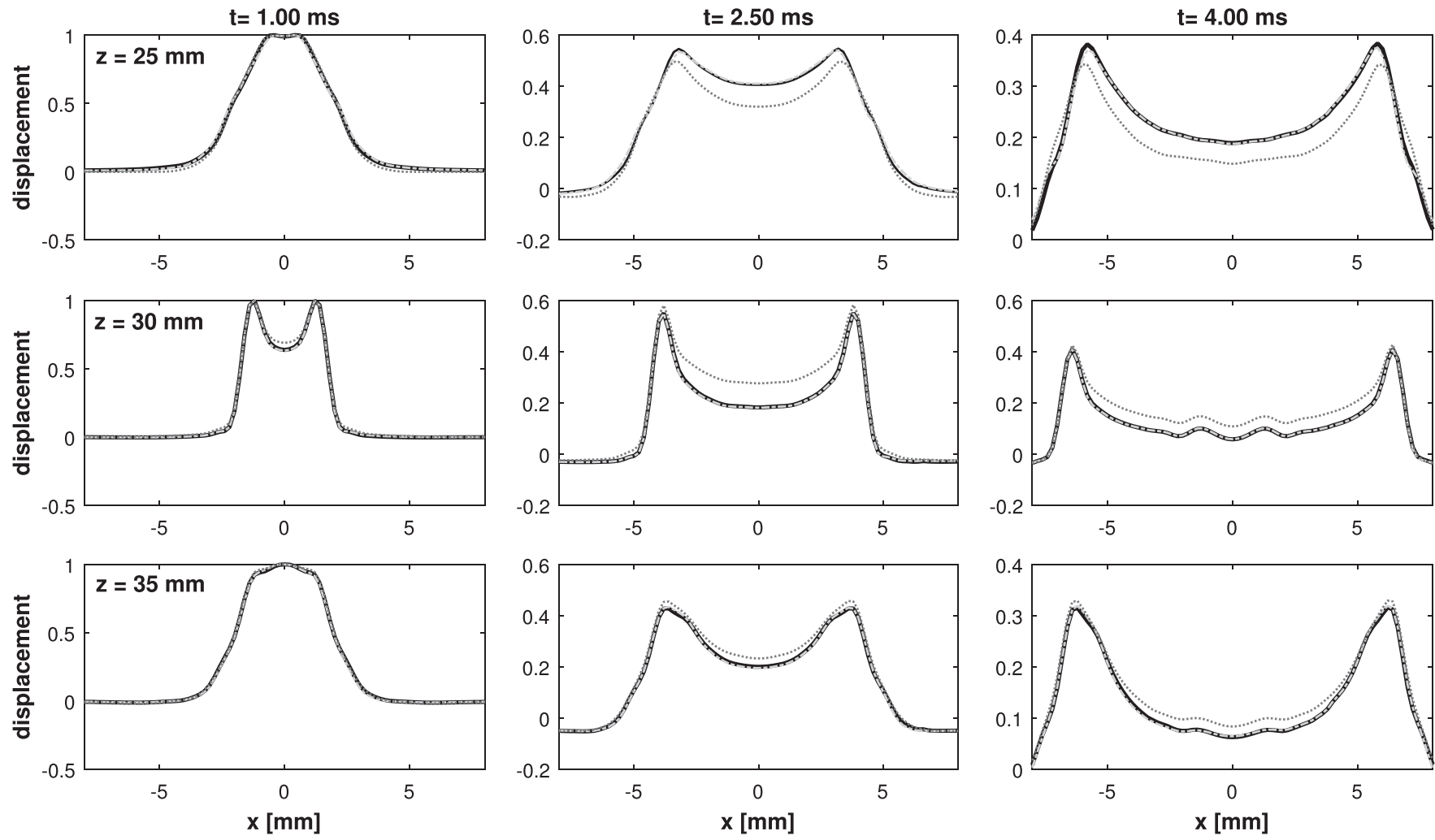

FIG. 10. Displacements in a homogeneous medium at $t=1 \mathrm{~ms}$ (left column), $t=2.5 \mathrm{~ms}$ (middle column), and $t=4 \mathrm{~ms}$ (right column) and at $25 \mathrm{~mm}$ depth (top row), $30 \mathrm{~mm}$ depth (middle row), and $35 \mathrm{~mm}$ depth (bottom row), when the ARF was computed using the second-order approximation (solid line), the quasi-plane wave approximation (dashed-dotted line), and the attenuated plane wave approximation (dotted line). In this case, each curve is normalized with the its maximum at $t=1$ ms.

The displacements computed using the quasi-plane wave approximation and the second-order approximation are nearly identical as expected and can hardly be distinguished.

To quantify the similarity between the displacements obtained with the second-order and the attenuated plane wave approximations a RMSE is computed. It is the root mean square of the difference between the displacement obtained using the ARF formulation with the second-order approximation and with the attenuated plane wave approximations.

The RMSE is below $2.3 \%$ at $t=1 \mathrm{~ms}$ but at $30 \mathrm{~mm}$ depth it grows to $11.1 \%$ and $11.2 \%$ at $t=2.5 \mathrm{~ms}$ and $t=4 \mathrm{~ms}$, respectively.

The displacements at points positioned on the axis, $1.5 \mathrm{~mm}$, and $3.0 \mathrm{~mm}$ off-axis, and at all three depths $(25,30$, and $35 \mathrm{~mm}$ ) are shown in Fig. 11.
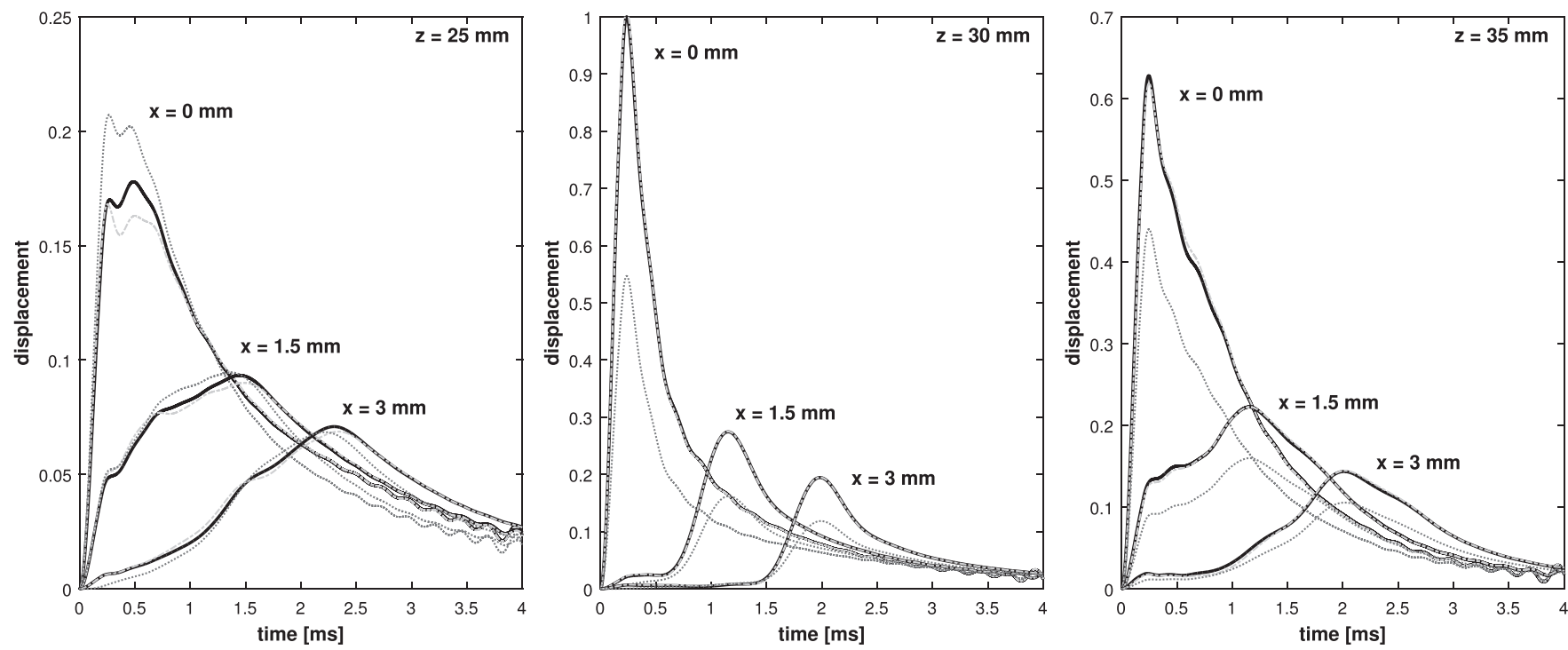

FIG. 11. Displacements in a homogeneous medium as a function of time for points positioned on the axis $(x=0 \mathrm{~mm}), 1.5 \mathrm{~mm}(x=1.5 \mathrm{~mm})$, and $3.0 \mathrm{~mm}$ $(x=3.0 \mathrm{~mm}$ ) off-axis and at $25 \mathrm{~mm}$ (left), $30 \mathrm{~mm}$ (middle), and $35 \mathrm{~mm}$ (right) depth. The displacements obtained using the second-order approximation, the quasi-plane wave approximation, and the attenuated plane wave approximation are shown in solid, dashed-dotted, and dotted lines, respectively. They are normalized with the maximum on-axis displacement computed at $30 \mathrm{~mm}$ depth using the second-order approximation. 
Again, they show that the displacements computed using the second-order approximation and the quasi-plane wave approximation are very similar. The minute differences that can be observed in Fig. 11 at $z=25 \mathrm{~mm}$ are attributed to numerical errors. On-axis, these displacements are slightly smaller than those computed with the attenuated plane wave approximation at $25 \mathrm{~mm}$ depth, but at 30 and $35 \mathrm{~mm}$ depth, they are much larger (approximately a factor 2 and 1.4, respectively). Similar trends can be observed at $x=1.5 \mathrm{~mm}$ and $x=3.0 \mathrm{~mm}$.

\section{DISCUSSION}

The analytical studies in the case of a spherical concave source and a quasi-Gaussian beam show clearly that there is a difference between the amplitude, and the direction of the ARF when computed using the second-order approximation and either the attenuated plane wave or the quasi-plane wave approximations.

The differences get larger as the source is more focused (larger values for $\theta$ or smaller values for $k a$ ). Indeed with a large focusing gain, the beam cannot be approximated to a plane or quasi-plane wave in most of the space due to the many propagation directions included in the beam. In that case both plane wave and quasi-plane wave approximations differ significantly from the second-order approximation.

An $F$-number of 1.5 or more is quite common for diagnostic probes. It is therefore reasonable in that case to use the quasi-plane wave approximation for estimating the ARF around focus. However, when using more focused transducers with lower $F$-numbers (below 1), only the secondorder approximation should be used.

When simulating the ARF and the ensuing shear displacement created by a diagnostic cardiac probe, there is a visible difference in the ARF field when using the three approximations (Fig. 8). However, there is a noticeable difference in the shear displacement only between the results obtained with the second-order approximation and the plane wave approximation. The displacements obtained using the second-order approximation and the quasi-plane wave approximation are nearly identical.

This demonstrates, as explained in Sec. V, that although the mean Eulerian excess pressure should be taken into account for a correct estimation of the ARF as in the secondorder approximation, it can be neglected for a prediction of the created shear displacement. The ARF expression in the quasi-plane wave or second-order approximation leads to identical theoretical displacements associated with the shear waves.

One limitation of the presented study is the formulation of the ARF based on a lossless stress tensor. Although the formulation accounts for momentum deposition due to attenuation and scattering from inhomogeneities it cannot account for losses due to large ruptures of acoustic impedance between tissue and bone, for instance. Forces and shear displacements due to the presence of strong inhomogeneities can be important and in some cases prevail over viscous forces. ${ }^{31}$ Likewise nonlinear propagation was neglected in this study, and in the case of high power ultrasound its contribution to ARF can be significant. ${ }^{32}$

\section{CONCLUSIONS}

We have presented three formulations of the ARF and the assumptions behind each of them. The second-order approximation does not assume anything on the geometry of the beam while the quasi-plane wave assumes that the beam is a bounded plane wave. The formulation of the ARF using the attenuated plane wave approximation and Eq. (18) for the expression of the intensity is a more coarse approach. It does not take into account the pressure or velocity spatial gradients, only the pressure amplitude. Because of that, it "flattens out" some spatial variations in the amplitude and direction of the ARF.

Analytical solutions for the ARF in the case of a concave spherical transducer and a quasi-Gaussian beam have shown the advantage to use the second-order approximation over the quasi-plane wave and attenuated plane wave approximations for highly focused sources or strongly divergent beams.

Numerical simulations for the ARF created by a diagnostic cardiac probe have shown the differences in the ARF computed when using the three approximations. The displacements generated by the ensuing shear wave, however, only differ between the second-order approximation and the attenuated plane wave approximation. This shows that the ARF expression using the second-order or quasi-plane wave approximations leads to identical displacements.

In conclusion we can state that to get an accurate estimate of the ARF the second-order approximation or the quasi-plane wave approximation should be used instead of the attenuated plane wave approximation for transducers with large $F$-numbers.

The part of the force in the second-order approximation formulation that is neglected in the quasi-plane wave approximation does not generate any shear motion in the tissue. In the context of SWEI both formulations can therefore be used equivalently to estimate the shear displacement created by the ARF.

\section{ACKNOWLEDGMENTS}

This research was supported by the Center for Innovative Ultrasound Solutions, the Research Council of Norway, Project Code 237887, and the Russian Foundation for Basic Research, Project No. 17-02-00261.

\section{APPENDIX A: EXPRESSION FOR THE ARF ON THE AXIS OF A SPHERICAL CONCAVE SOURCE USING THE SECOND-ORDER APPROXIMATION}

On the axis of an axisymmetric source due to symmetry the ARF is directed along the axis and only the $z$ component of the ARF is not null. For the second-order approximation formulation we have according to Sec. III A,

$$
F_{z}(0,0, z)=\frac{j \alpha(\omega)}{2 \omega \rho_{0} c_{0}}\left(P \frac{\partial P^{*}}{\partial z}-P^{*} \frac{\partial P}{\partial z}\right) .
$$


We aim at finding an expression for Eq. (A1) in our particular case of a spherical concave source.

On the axis the expression of the complex pressure amplitude is

$$
P(0,0, z)=\rho_{0} c_{0} V_{0} \frac{e^{j k z}-e^{j k R_{\max }}}{1-\frac{z}{R}} .
$$

Let us compute $P\left(\partial P^{*} / \partial z\right)$.

First

$$
\begin{aligned}
\frac{\partial P}{\partial z}= & \frac{\rho_{0} c_{0} V_{0}}{\left(1-\frac{z}{R}\right)^{2}}\left[\left(1-\frac{z}{R}\right)\left(j k e^{j k z}-j k R_{\max }^{\prime} e^{j k R_{\max }}\right)\right. \\
& \left.+\frac{1}{R}\left(e^{j k z}-e^{j k R_{\max }}\right)\right],
\end{aligned}
$$

where

$$
R_{\max }^{\prime}=\frac{\partial R_{\max }}{\partial z}=\frac{\frac{z}{R}-1+\cos \theta}{\sqrt{1+\left(1-\frac{z}{R}\right)^{2}-2\left(1-\frac{z}{R}\right) \cos \theta}} .
$$

Then

$$
\begin{aligned}
P \frac{\partial P^{*}}{\partial z}= & \frac{\left(\rho_{0} c_{0} V_{0}\right)^{2}}{\left(1-\frac{z}{R}\right)^{3}}\left(e^{j k z}-e^{j k R_{\max }}\right)\left[\left(1-\frac{z}{R}\right)\right. \\
& \times\left(-j k e^{-j k z}+j k R_{\max }^{\prime} e^{-j k R_{\max }}\right) \\
& \left.+\frac{1}{R}\left(e^{-j k z}-e^{-j k R_{\max }}\right)\right]
\end{aligned}
$$

and

$$
\begin{aligned}
P \frac{\partial P^{*}}{\partial z}-P^{*} \frac{\partial P}{\partial z}= & -\frac{j k\left(\rho_{0} c_{0} V_{0}\right)^{2}}{\left(1-\frac{z}{R}\right)^{2}} 2\left(R_{\max }^{\prime}+1\right) \\
& \times\left[1-\cos \left(k\left(z-R_{\max }\right)\right)\right] .
\end{aligned}
$$

The expression of the ARF on axis then becomes

$$
F_{z}(0,0, z)=\frac{\alpha(\omega) P_{0}^{2}}{\rho_{0} c_{0}^{2}} \frac{\left(R_{\max }^{\prime}+1\right)}{\left(1-\frac{z}{R}\right)^{2}}\left[1-\cos \left(\frac{\omega}{c_{0}}\left(z-R_{\max }\right)\right)\right],
$$

where

$$
P_{0}=\rho_{0} c_{0} V_{0} .
$$

\section{APPENDIX B: EXPRESSION OF THE ARF ON THE AXIS OF A SPHERICAL CONCAVE SOURCE USING THE QUASI-PLANE WAVE APPROXIMATION}

According to Eq. (38)

$$
F_{z}(0,0, z)=\frac{j \omega A}{4 c_{0}^{2}} P^{*} \frac{\partial P}{\partial z}-\frac{\rho_{0} A A^{*}}{4} \frac{\partial^{2} P}{\partial z^{2}} \frac{\partial P^{*}}{\partial z}+\text { c.c. }
$$

The expressions for $P^{*}(\partial P / \partial P)$ and $\partial P^{*} / \partial z$ were established in Appendix A. We therefore only need to establish an expression for $\partial^{2} P / \partial z^{2}$ :

$$
\begin{aligned}
\frac{\partial^{2} P}{\partial z^{2}}= & P_{0}\left[\frac{-k^{2} e^{j k z}-j k R_{\max }^{\prime \prime} e^{j k R_{\max }}+k^{2} R_{\max }^{\prime 2} e^{j k R_{\max }}}{1-\frac{z}{R}}\right. \\
& \left.+2 \frac{j k e^{j k z}-j k R_{\max }^{\prime} e^{j k R_{\max }}}{R\left(1-\frac{z}{R}\right)^{2}}+2 \frac{e^{j k z}-e^{j k R_{\max }}}{R^{2}\left(1-\frac{z}{R}\right)^{3}}\right]
\end{aligned}
$$

where

$$
R_{\max }^{\prime \prime}=\frac{\partial^{2} R_{\max }}{\partial z^{2}}=\frac{(R \sin \theta)^{2}}{R_{\max }^{3}}
$$

Combining Eqs. (A2), (A3), and the above equations, we find an analytic expression for $F_{z}(0,0, z)$ on the axis of the source for a spherical concave source. The expression is quite large and is not given here for brevity.

\section{APPENDIX C: EXPRESSION OF THE ARF FOR A QUASI-GAUSSIAN BEAM}

When using the second-order approximation Eq. (27) is used while when using the quasi-plane wave approximation Eq. (32) is used.

We therefore need to establish the expression for the first and second spatial derivatives of the pressure.

Expressions anywhere in space can be obtained but we will derive here the above expressions in the plane $y=0$. Because of symmetry of the field, all components in $y$ will therefore be zero in this plane.

We are therefore looking for an expression for $F_{x}(x, 0$, $z)$ and $F_{z}(x, 0, z)$, the radial and longitudinal components of the ARF.

In the following derivations since $x$ and $y$ play a symmetric role only expressions involving the variables $x$ and $z$ are shown. Expressions involving $y$ can be deduced by switching $x$ with $y$.

Let us first define

$$
B=\frac{P_{0} z_{d}}{2 \sinh ^{2}\left(k z_{d}\right)},
$$

so that the expression for the complex pressure amplitude is

$$
P(x, y, z)=B\left[e^{k z_{d}} \frac{\sin \left(k \sqrt{D_{-}}\right)}{\sqrt{D_{-}}}-e^{-k z_{d}} \frac{\sin \left(k \sqrt{D_{+}}\right)}{\sqrt{D_{+}}}\right]
$$

Remembering that $D_{-}=x^{2}+y^{2}+\left(z-j z_{d}\right)^{2}$ and $D_{+}=x^{2}$ $+y^{2}+\left(z+j z_{d}\right)^{2}$ we get the following expression for the spatial derivatives of $P$ : 


$$
\frac{\partial P}{\partial z}=B\left[e^{k z_{d}}\left(\frac{k\left(z-j z_{d}\right) \cos \left(k \sqrt{D_{-}}\right)}{D_{-}}-\frac{\left(z-j z_{d}\right) \sin \left(k \sqrt{D_{-}}\right)}{D_{-}^{3 / 2}}\right)-e^{-k z_{d}}\left(\frac{k\left(z+j z_{d}\right) \cos \left(k \sqrt{D_{+}}\right)}{D_{+}}-\frac{\left(z+j z_{d}\right) \sin \left(k \sqrt{D_{+}}\right)}{D_{+}^{3 / 2}}\right)\right] .
$$

By replacing both $\left(z-j z_{d}\right)$ and $\left(z+j z_{d}\right)$ with $x$ or $y$ we get similar expressions for $\partial P / \partial x$ or $\partial P / \partial y$, respectively.

We then evaluate the second spatial derivatives

$$
\begin{aligned}
\frac{\partial^{2} P}{\partial z^{2}}= & B\left[e^{k z_{d}}\left(\frac{k \cos \left(k \sqrt{D_{-}}\right)}{D_{-}}-\left(1+k^{2}\left(z-j z_{d}\right)^{2}\right) \frac{\sin \left(k \sqrt{D_{-}}\right)}{D_{-}^{3 / 2}}-3 k\left(z-j z_{d}\right)^{2} \frac{\cos \left(k \sqrt{D_{-}}\right)}{D_{-}^{2}}+3\left(z-j z_{d}\right)^{2} \frac{\sin \left(k \sqrt{D_{-}}\right)}{D_{-}^{5 / 2}}\right)\right. \\
& \left.-e^{-k z_{d}}\left(\frac{k \cos \left(k \sqrt{D_{+}}\right)}{D_{+}}-\left(1+k^{2}\left(z+j z_{d}\right)^{2}\right) \frac{\sin \left(k \sqrt{D_{+}}\right)}{D_{+}^{3 / 2}}-3 k\left(z+j z_{d}\right)^{2} \frac{\cos \left(k \sqrt{D_{+}}\right)}{D_{+}^{2}}+3\left(z+j z_{d}\right)^{2} \frac{\sin \left(k \sqrt{D_{+}}\right)}{D_{+}^{5 / 2}}\right)\right] .
\end{aligned}
$$

Again, by replacing both $\left(z-j z_{d}\right)$ and $\left(z+j z_{d}\right)$ with $x$ or $y$ we get similar expressions for $\partial^{2} P / \partial x^{2}$ or $\partial^{2} P / \partial y^{2}$, respectively.

$$
\begin{aligned}
\frac{\partial^{2} P}{\partial x \partial z}= & B\left[e^{k z_{d}}\left(-k^{2} x\left(z-j z_{d}\right) \frac{\sin \left(k \sqrt{D_{-}}\right)}{D_{-}^{3 / 2}}-3 k x\left(z-j z_{d}\right) \frac{\cos \left(k \sqrt{D_{-}}\right)}{D_{-}^{2}}+3 x\left(z-j z_{d}\right) \frac{\sin \left(k \sqrt{D_{-}}\right)}{D_{-}^{5 / 2}}\right)\right. \\
& \left.-e^{-k z_{d}}\left(-k^{2} x\left(z+j z_{d}\right) \frac{\sin \left(k \sqrt{D_{+}}\right)}{D_{+}^{3 / 2}}-3 k x\left(z+j z_{d}\right) \frac{\cos \left(k \sqrt{D_{+}}\right)}{D_{+}^{2}}+3 x\left(z+j z_{d}\right) \frac{\sin \left(k \sqrt{D_{+}}\right)}{D_{+}^{5 / 2}}\right)\right] .
\end{aligned}
$$

And finally by replacing $x$ with $y$ in the above expression we get an expression for $\partial^{2} P / \partial y \partial z$ and by replacing both $\left(z-j z_{d}\right)$ and $\left(z+j z_{d}\right)$ with $y$ we get an expression for $\partial^{2} P / \partial x \partial y$.

From these equations we can get an analytical expressions for $F_{x}(x, 0, z)$ and $F_{z}(x, 0, z)$ using the second-order or the quasi-plane wave approximation. The expressions are quite large and will not be shown here for brevity.

${ }^{1}$ J.-L. Gennisson, T. Deffieux, M. Fink, and M. Tanter, "Ultrasound elastography: Principles and techniques,” Diagn. Interv. Imag. 94(5), 487-495 (2013).

${ }^{2}$ J. R. Doherty, G. E. Trahey, K. R. Nightingale, and M. L. Palmeri, "Acoustic radiation force elasticity imaging in diagnostic ultrasound," IEEE Trans. Ultrason. Ferroelectr. Freq. Control 60(4), 685-701 (2013).

${ }^{3}$ R. Sinkus, M. Tanter, T. Xydeas, S. Catheline, J. Bercoff, and M. Fink, "Viscoelastic shear properties of in vivo breast lesions measured by $\mathrm{mr}$ elastography," Magn. Reson. Imag. 23(2), 159-165 (2005).

${ }^{4}$ E. E. Konofagou, C. Maleke, and J. Vappou, "Harmonic motion imaging (HMI) for tumor imaging and treatment monitoring," Curr. Med. Imag. Rev. 8(1), 16-26 (2012).

${ }^{5}$ A. P. Sarvazyan, O. V. Rudenko, and W. L. Nyborg, "Biomedical applications of radiation force of ultrasound: Historical roots and physical basis," Ultrasound Med. Biol. 36(9), 1379-1394 (2010).

${ }^{6}$ M. L. Palmeri, A. C. Sharma, R. R. Bouchard, R. W. Nightingale, and K. R. Nightingale, "A finite-element method model of soft tissue response to impulsive acoustic radiation force," IEEE Trans. Ultrason. Ferroelectr. Freq. Control 52(10), 1699-1712 (2005).

${ }^{7}$ K. R. Nightingale, R. W. Nightingale, M. L. Palmeri, and G. E. Trahey, "A finite element model of remote palpation of breast lesions using radiation force: Factors affecting tissue displacement," Ultrasonic Imag. 22(1), 35-54 (2000).

${ }^{8}$ S. Callé, J.-P. Remeniéras, O. Bou Matar, M. E. Hachemi, and F. Patat, "Temporal analysis of tissue displacement induced by a transient ultrasound radiation force," J. Acoust. Soc. Am. 118(5), 2829-2840 (2005).
${ }^{9}$ C. Bastard, J.-P. Remeniéras, S. Callé, and L. Sandrin, "Simulation of shear wave propagation in a soft medium using a pseudospectral time domain method," J. Acoust. Soc. Am. 126(4), 2108-2116 (2009).

${ }^{10}$ L. D. Landau and E. M. Lifshitz, "Ideal fluids," in Fluid Mechanics, Vol. 6 of Course of Theoretical Physics, 2nd ed. (Pergamon Press, Oxford, England, 1987), Chap. 1, pp. 1-43.

${ }^{11}$ C. P. Lee and T. G. Wang, "Acoustic radiation pressure," J. Acoust. Soc. Am. 94(2), 1099-1109 (1993).

${ }^{12}$ S. D. Danilov and M. A. Mironov, "Mean force on a small sphere in a sound field in a viscous fluid," J. Acoust. Soc. Am. 107(1), 143-153 (2000).

${ }^{13}$ T. Hasegawa, T. Kido, T. Iizuka, and C. Matsuoka, "A general theory of Rayleigh and Langevin radiation pressures,” J. Acoust. Soc. Jpn. 21(3), 145-152 (2000).

${ }^{14}$ W. L. Nyborg, "Acoustic streaming," in Physical Acoustics, edited by W. P. Mason (Academic Press, New York, 1965), Vol. 2 Pt. B, Chap. 11, pp. $265-331$.

${ }^{15}$ P. Glynne-Jones, P. P. Mishra, R. J. Boltryk, and M. Hill, "Efficient finite element modeling of radiation forces on elastic particles of arbitrary size and geometry," J. Acoust. Soc. Am. 133(4), 1885-1893 (2013).

${ }^{16}$ O. A. Sapozhnikov and M. R. Bailey, "Radiation force of an arbitrary acoustic beam on an elastic sphere in a fluid," J. Acoust. Soc. Am. 133(2), 661-676 (2013).

${ }^{17}$ H. Saraf, K. T. Ramesh, A. M. Lennon, A. C. Merkle, and J. C. Roberts, "Mechanical properties of soft human tissues under dynamic loading," J. Biomech. 40(9), 1960-1967 (2007).

${ }^{18}$ H. C. Starritt, F. A. Duck, and V. F. Humphrey, "Forces acting in the direction of propagation in pulsed ultrasound fields," Phys. Med. Biol. 36(11), 1465-1474 (1991).

${ }^{19}$ K. R. Nightingale, M. L. Palmeri, R. W. Nightingale, and G. E. Trahey, "On the feasibility of remote palpation using acoustic radiation force," J. Acoust. Soc. Am. 110(1), 625-634 (2001).

${ }^{20}$ O. A. Sapozhnikov, "An exact solution to the Helmholtz equation for a quasi-Gaussian beam in the form of a superposition of two sources and sinks with complex coordinates," Acoust. Phys. 58, 41-47 (2012).

${ }^{21}$ H. T. O'Neil, "Theory of focusing radiators," J. Acoust. Soc. Am. 21(5), 516-526 (1949).

${ }^{22}$ P. L. Marston, "Quasi-Gaussian Bessel-beam superposition: Application to the scattering of focused waves by spheres," J. Acoust. Soc. Am. 129(4), 1773-1782 (2011). 
${ }^{23}$ P. L. Marston, "Quasi-Gaussian beam analytical basis and comparison with an alternative approach (L)," J. Acoust. Soc. Am. 130(3), 1091-1094 (2011). ${ }^{24}$ J. Lighthill, "Acoustic streaming," J. Sound Vib. 61(3), 391-418 (1978).

${ }^{25}$ O. A. Sapozhnikov, "High-intensity ultrasonic waves in fluids: Nonlinear propagation and effects," in Power Ultrasonics: Applications of High-intensity Ultrasound, No. 66 in Series in Electronic and Optical Materials, edited by J. A. Gallego Juárez and K. F. Graff (Woodhead Publishing, Cambridge, UK, 2015), Chap. 2, pp. 9-32.

${ }^{26}$ B. E. Treeby, J. Jaros, A. P. Rendell, and B. T. Cox, "Modeling nonlinear ultrasound propagation in heterogeneous media with power law absorption using a k-space pseudospectral method," J. Acoust. Soc. Am. 131(6), 4324-4336 (2012).

${ }^{27}$ F. Prieur and S. Catheline, "Simulation of shear wave elastography imaging using the toolbox 'k-Wave,", in Proceedings of the 172nd Meeting Acoustical Society of America, Vol. 29, Honolulu, HI (December 2016), p. 020002 .
${ }^{28}$ B. E. Treeby, J. Jaros, D. Rohrbach, and B. Cox, "Modelling elastic wave propagation using the k-Wave Matlab toolbox," in Proceedings of the IEEE Ultrasonic Symposium, Chicago, IL (2014), pp. 146-149.

${ }^{29}$ K. Firouzi, B. T. Cox, B. E. Treeby, and N. Saffary, "A first-order $k$-space model for elastic wave propagation in heterogeneous media," J. Acoust. Soc. Am. 132(3), 1271-1283 (2012).

${ }^{30} \mathrm{~B}$. Treeby, B. Cox, and J. Jaros, k-Wave A MATLAB Toolbox for the Time Domain Simulation of Acoustic Wave Fields-User Manual, November 2012. Manual Version 1.0.1 http://www.k-wave.org/manual/kwave_user_manual_1.0.1.pdf (Last viewed July 10, 2017).

${ }^{31}$ L. Ostrovsky, A. Sutin, Y. Il'inskii, O. Rudenko, and A. Sarvazyan, "Radiation force and shear motions in inhomogeneous media," J. Acoust. Soc. Am. 121(3), 1324-1331 (2007).

${ }^{32}$ O. V. Rudenko, A. P. Sarvazyan, and S. Y. Emelianov, "Acoustic radiation force and streaming induced by focused nonlinear ultrasound in a dissipative medium,” J. Acoust. Soc. Am. 99(5), 2791-2798 (1996). 